\title{
Forecasting of the Upper Atmosphere via Assimilation of Electron Density Data
}

Timothy Kodikara
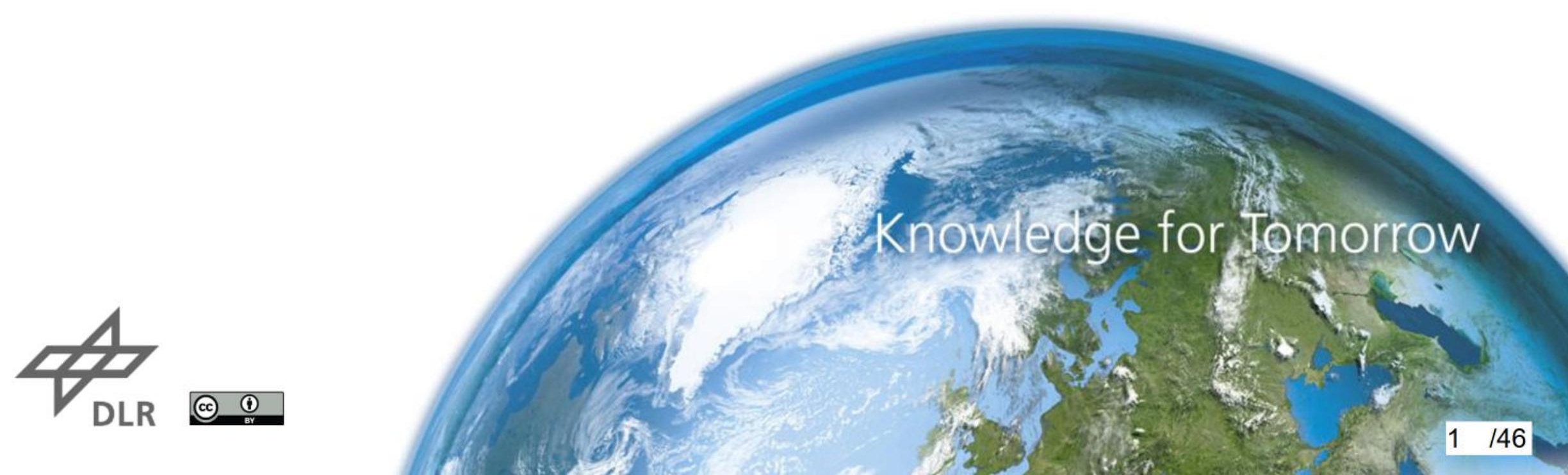


\section{Outline}

- The Study

- TIE-GCM

- Data Assimilation

- Results

- Validation of the Forecasts

- Impact on the Thermosphere

- Summary and Conclusions 


\section{The Study}

This study presents results from experiments of driving a physics-based thermosphere model (TIE-GCM) by assimilating radio occultation electron density $(\mathrm{Ne})$ profiles from the joint USA-Taiwan Constellation Observing System for Meteorology, lonosphere and Climate/Formosa Satellite 3 (COSMIC/FORMOSAT-3; hereinafter COSMIC) mission using an ensemble Kalman filter.

This study not only helps to gauge the accuracy of the assimilation, to explain the inherent model bias, and to understand the limitations of the framework, but it also demonstrates the capability of the assimilation technique to forecast the highly dynamical thermosphere in the presence of realistic data assimilation scenarios. 


\section{Thermosphere lonosphere Electrodynamics-General Circulation Model}

Magnetic activity High-lat input

\section{(Kp, IMF)}

Heelis et al.. 1982:

Weimer 2005;

AMIE (data assimilation);

LFM magnetosphere

Low/mid lat electrodynamics: neutral wind dynamo in geo-mag apex coordinates. Richmond et al., 1992; 1995

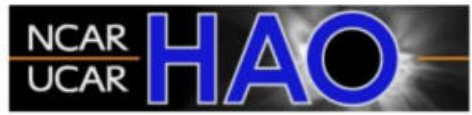

Developed by the High Altitude Observatory, Colorado, USA
Solar EUV/FUV Lower atm forcing (tides) $\left(F_{10.7}\right)$

$\mathrm{F}_{10.7}$ based EUVAC; Solomon \& Qian, 2005;
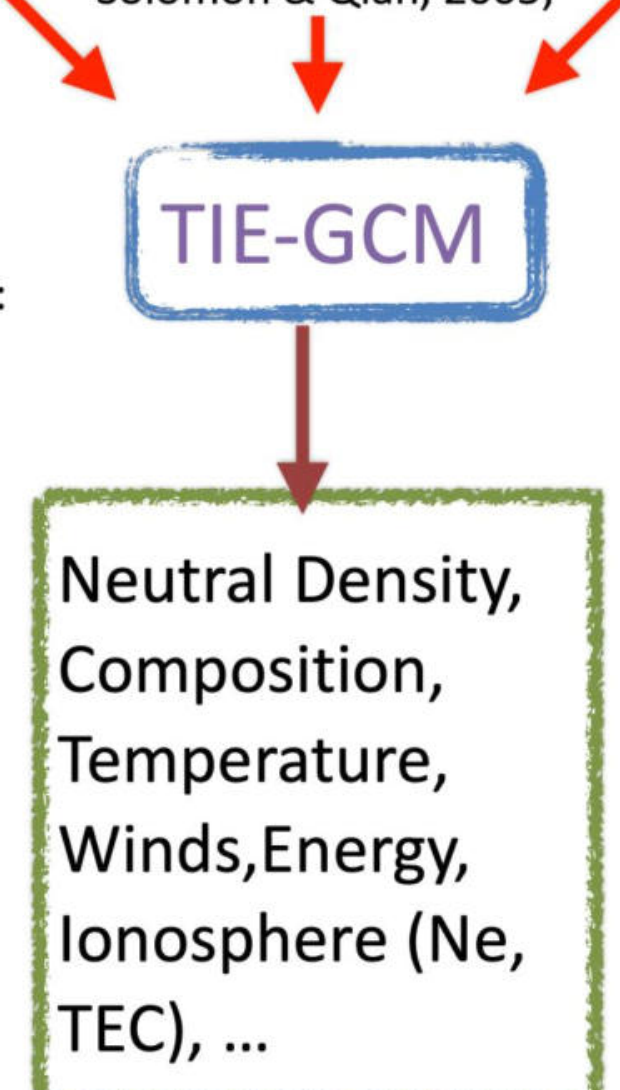

Latitude $x$ Longitude: $2.5^{\circ} \times 2.5^{\circ}$ or $5^{\circ} \times 5^{\circ}$ Height: $\underline{29}$ or 57 constant pressure levels
Configuration used in this study
GSWM, Hagan et al., 1999 Eddy, Qian et al., 2009

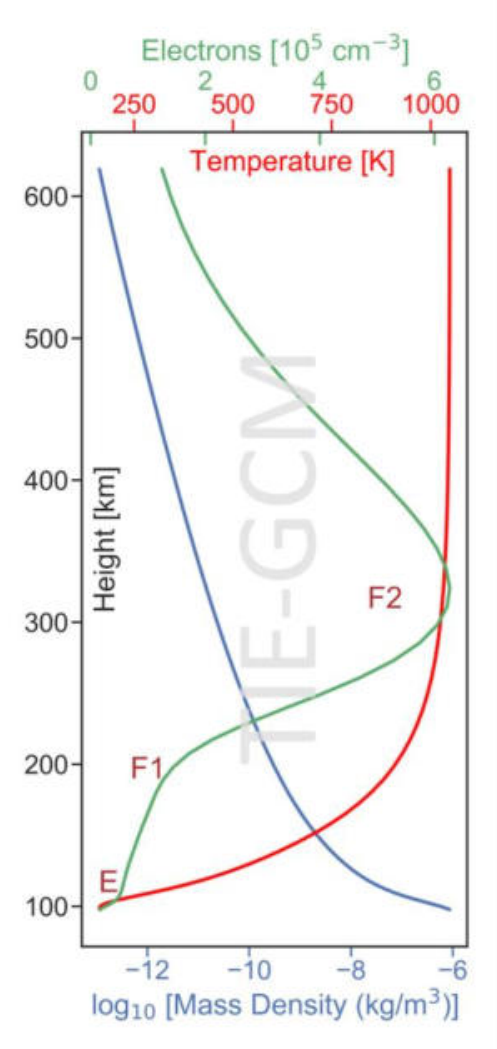




\section{Data Assimilation - Ensemble Kalman Filter}

NCAR's

Data

Assimilation

Research

Testbed

DART parameters (ensemble size, localisation, assimilation window,...)

Assimilate the obs using the EnKF

State vector

$\boldsymbol{x}=\left[\boldsymbol{\psi}^{T n} ; \boldsymbol{\psi}^{\gamma \mathrm{O}} ; \boldsymbol{\psi}^{\gamma \mathrm{O}^{+}} ; \boldsymbol{\psi}^{\gamma \mathrm{O}_{2}} ; \boldsymbol{\psi}^{U} ; \boldsymbol{\psi}^{V} ; \boldsymbol{\psi}^{\mathrm{Ne}}\right]$

Advance the model

Diagnostics: explore state before and after assimilation cycle

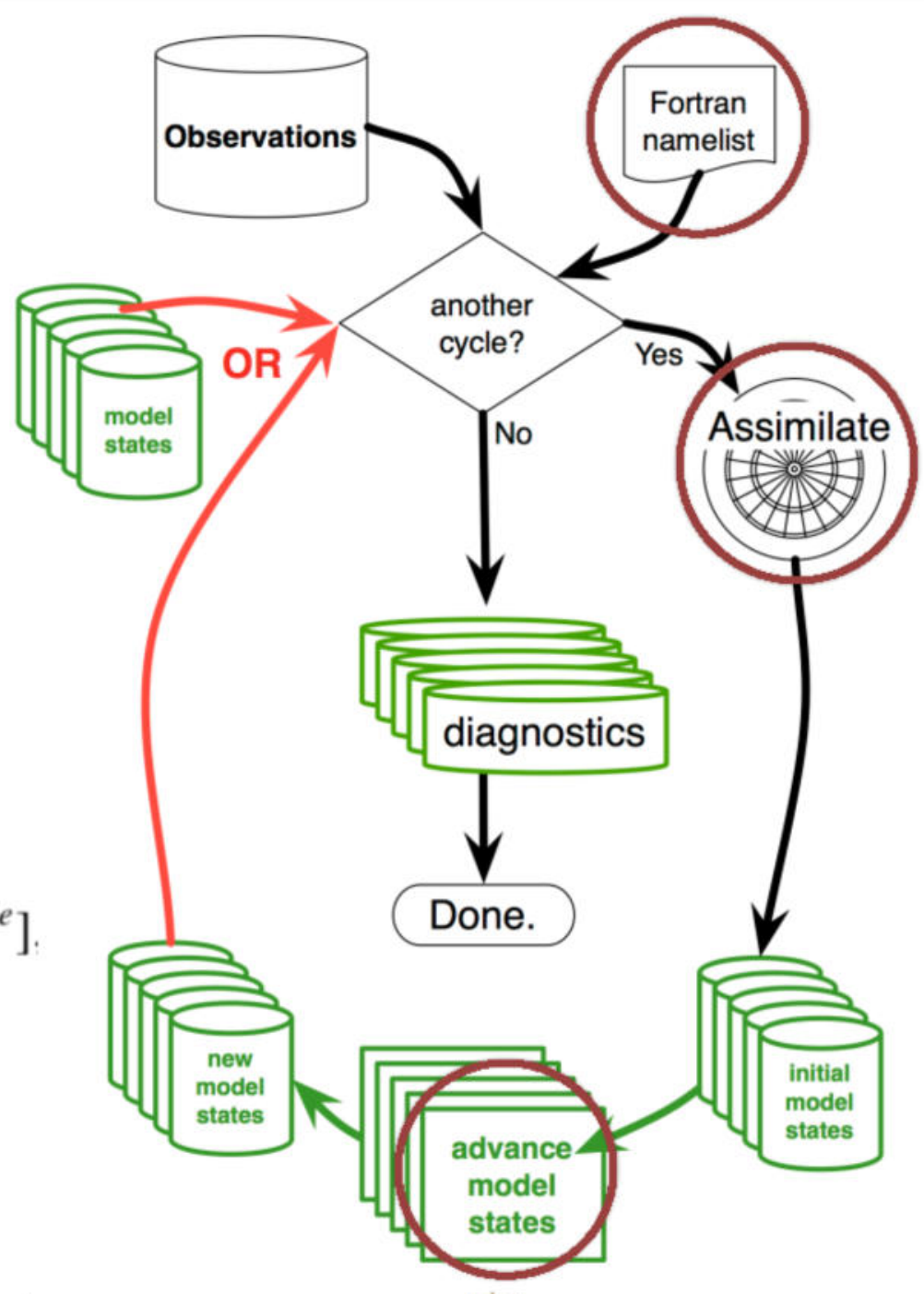

Integrated the latest TIE-GCM into DART to perform these experiments

Credit: NCAR 


\section{Results from Experiments E1 and E2}

D E1: Assimilate COSMIC-Ne during solar minimum (2008 March 4-8)

> E2: Assimilate COSMIC-Ne during solar maximum (2014 June 2-6) 


\section{Description of the next three figures:}

The number of COSMIC-Ne profiles available to assimilate significantly depends on latitude and local time. The following three figures show the root mean square error (RMSE) for E1 relative to COSMIC-Ne observations at six different altitude regions with a width of $50 \mathrm{~km}$ extending from 150 to $450 \mathrm{~km}$. Each Figure is for a specific latitude region. The results are averaged hourly by local time (LT).

The number of assimilated observations per LT-bin is marked in green. The gaps in the lines indicate the unavailability of observations to represent the respective LT in the specified latitude-altitude region. The TIE-GCM run is driven by using daily geophysical indices (F10.7, Kp). The forecast here is a forecast with a lead time of one hour. The analysis is the state after assimilation. As the assimilation progresses, the forecast run uses the analysis state from the previous time step to compute the expected state of the current time step. For example, the forecast state at 2 UT used the analysis state at 1 UT as the base state to drive the model forward by 1 hour. 
E1 RMSE evolution of Ne relative to COSMIC data
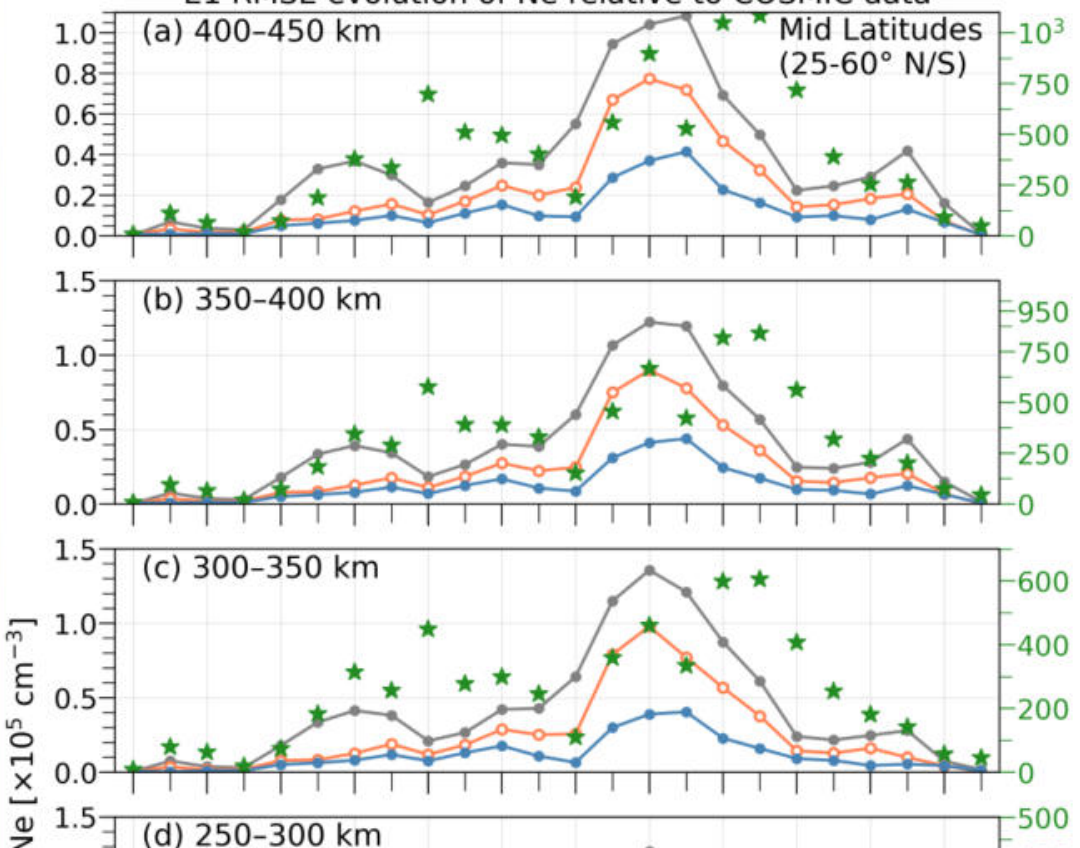

这
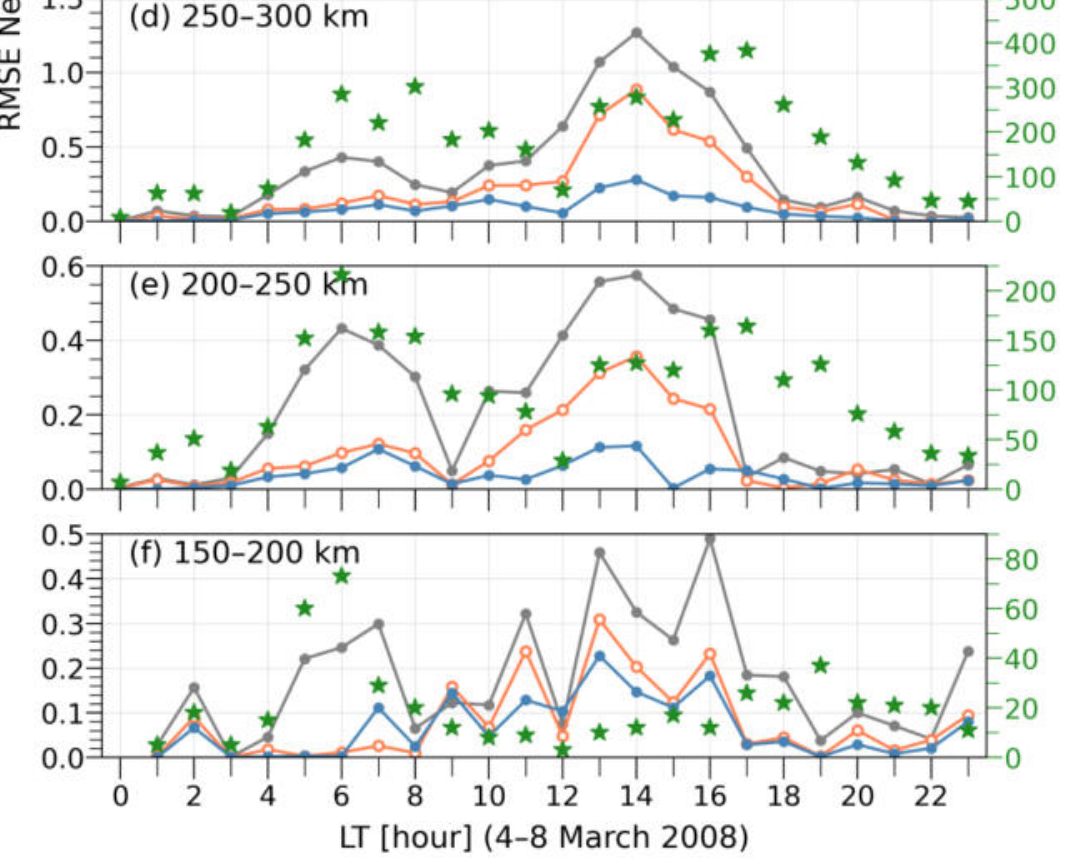

LT [hour] (4-8 March 2008)

- TIE-GCM - 0 Forecast $\bullet$ Analysis 
E1 RMSE evolution of Ne relative to COSMIC data
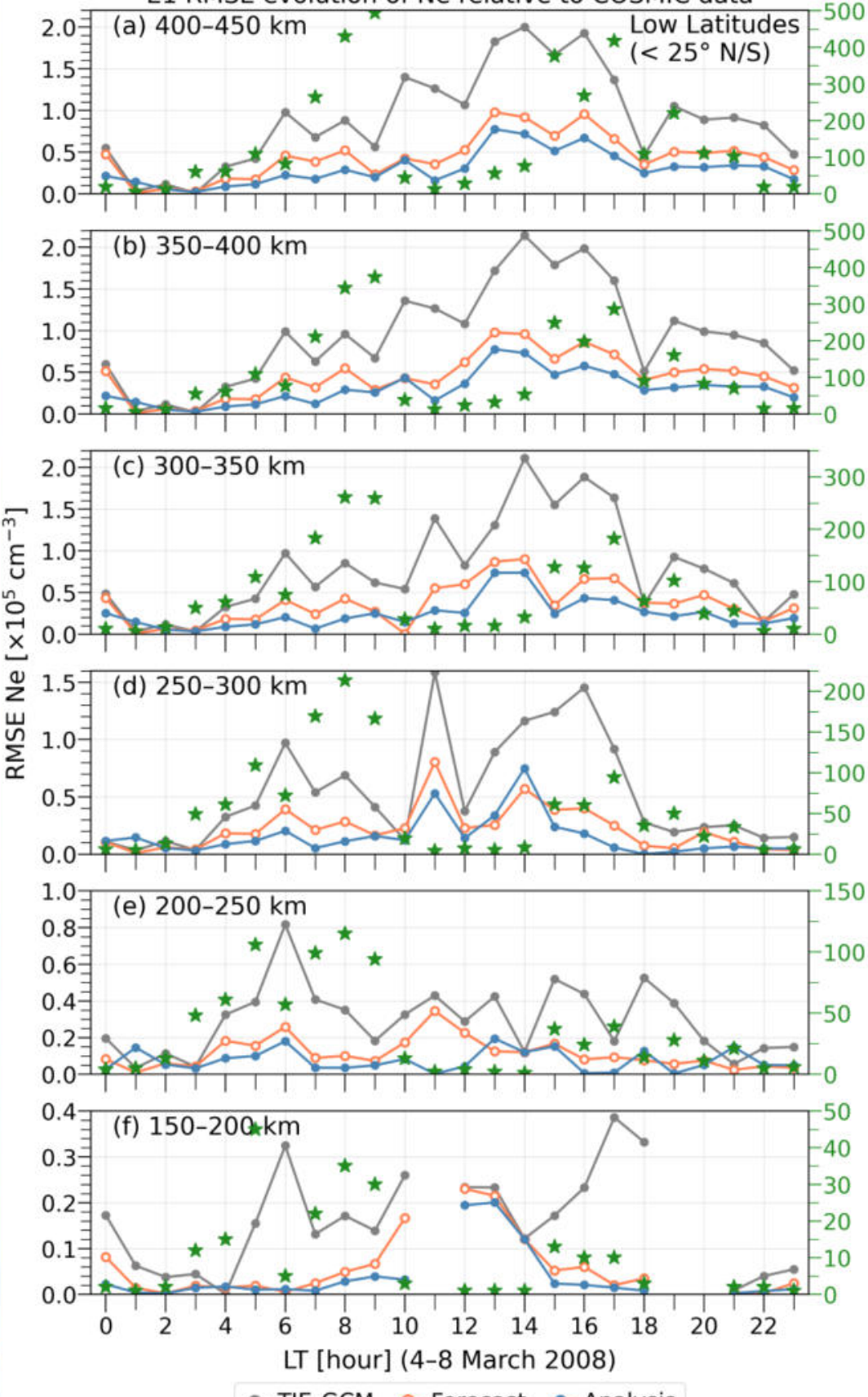
E1 RMSE evolution of Ne relative to COSMIC data
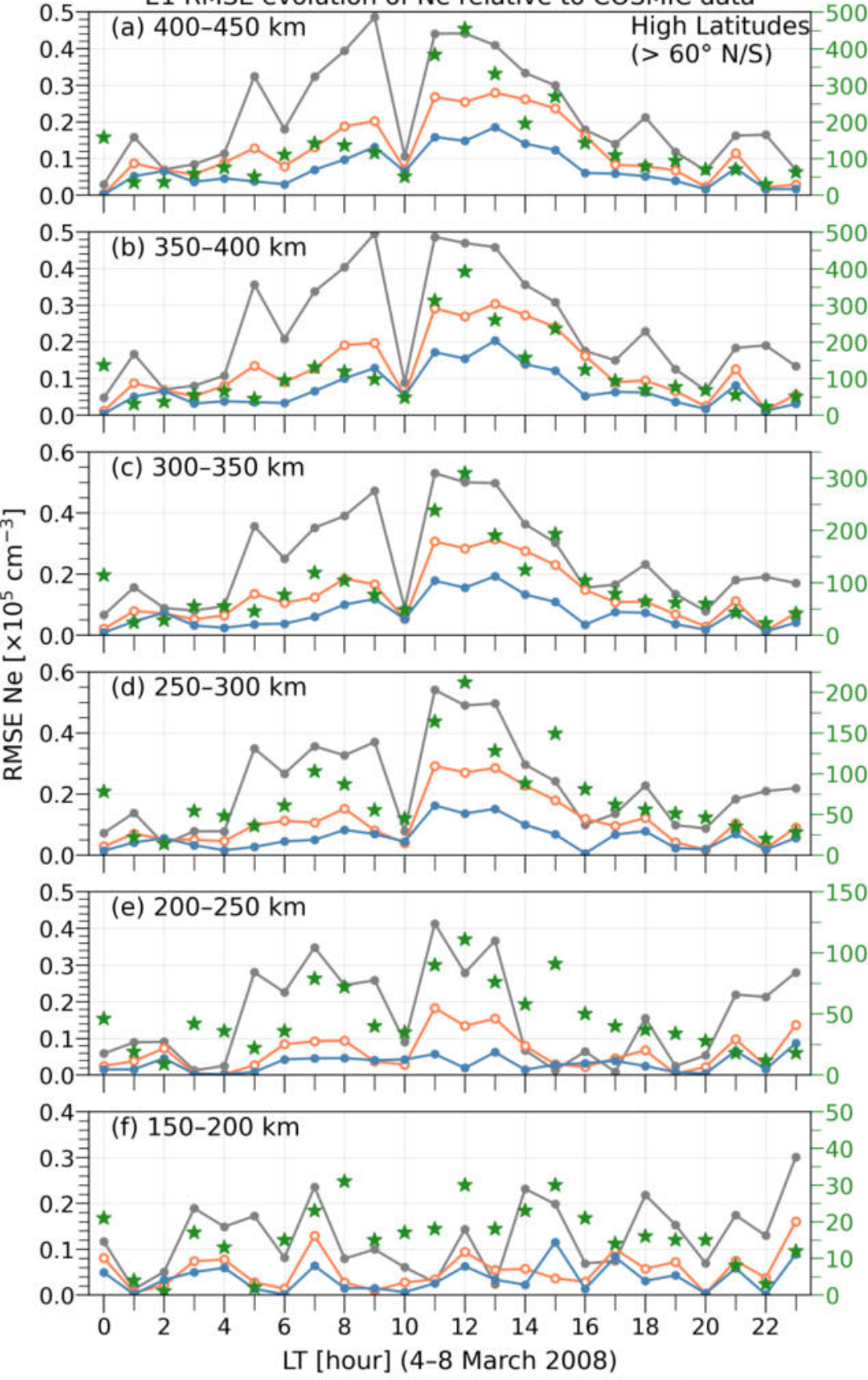

LT [hour] (4-8 March 2008)

- TIE-GCM -o- Forecast * Analysis 
The following three figures are similar to the previous three figures except for E2. 
E2 RMSE evolution of Ne relative to COSMIC data
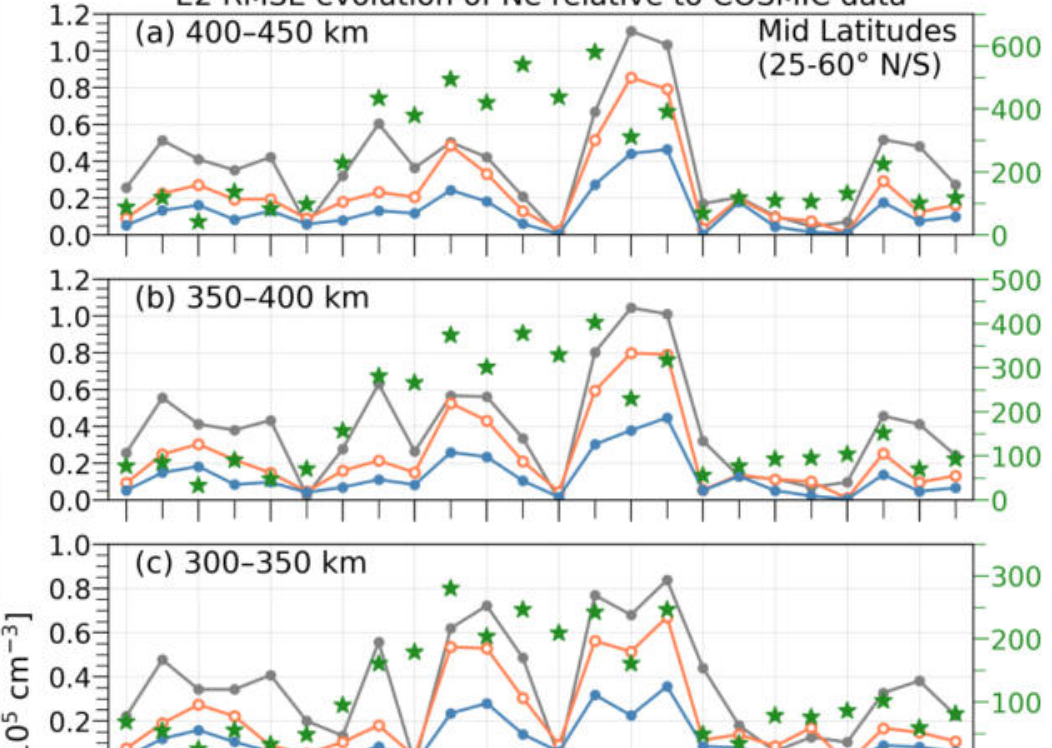

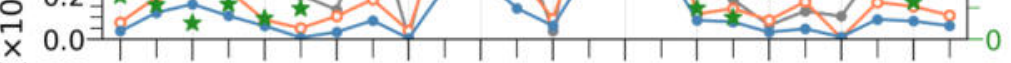

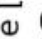

(d) $250-300 \mathrm{~km}$ औ

山 0.6

$\sum_{\simeq} 0.4 \exists$

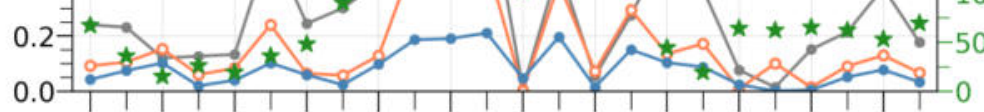
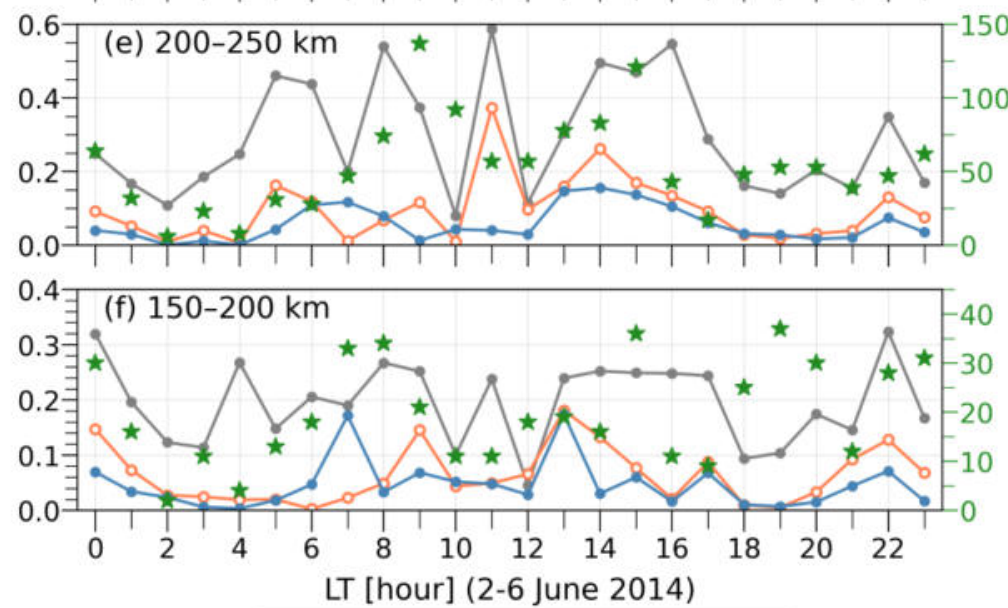

LT [hour] (2-6 June 2014)

- TIE-GCM - - Forecast * Analysis 
E2 RMSE evolution of Ne relative to COSMIC data
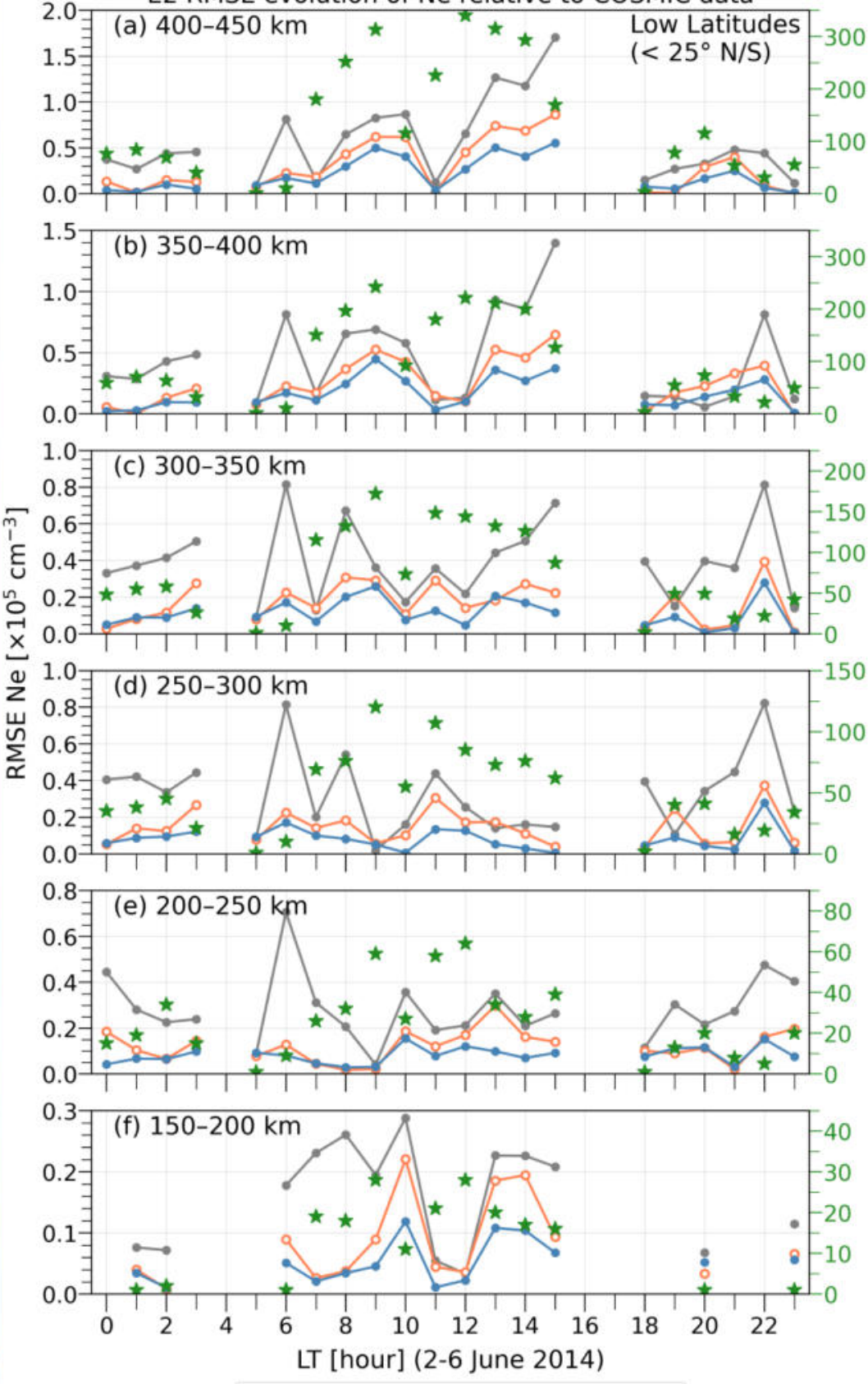

- TIE-GCM - Forecast * Analysis 
E2 RMSE evolution of Ne relative to COSMIC data

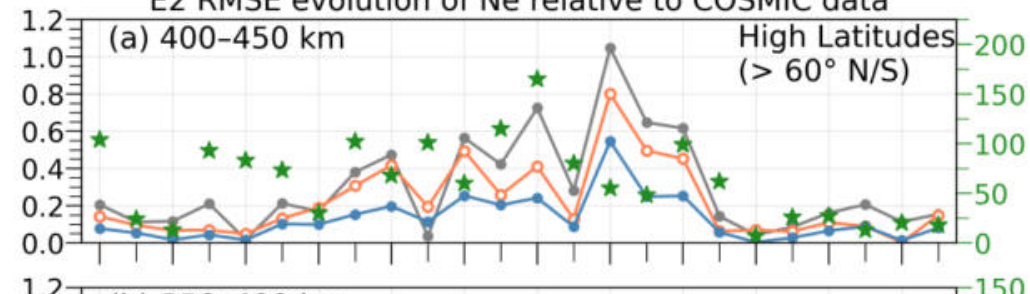

1.2 (b) $350-400 \mathrm{~km}$

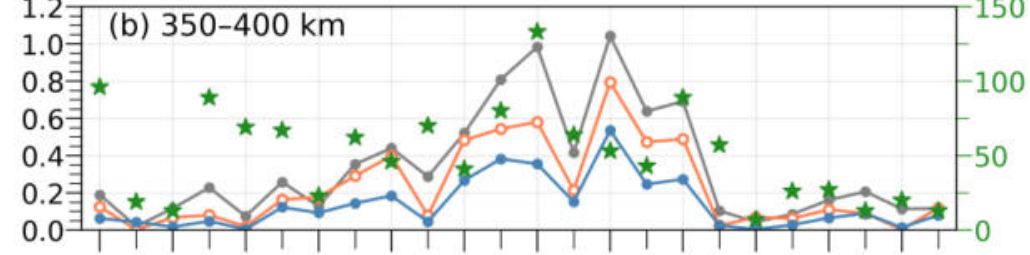

$1.5 \Rightarrow$ (c) $300-350 \mathrm{~km}$

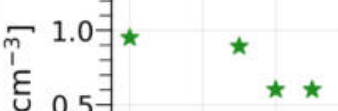

0.5

ํำ

$\times 0.0$ | | | | | | | | | | | | | | | | | | | | | 0

$\stackrel{0}{z} 1.5$ (d) $250-300 \mathrm{~km}$

岕 $1.0=$ *

$\sum_{\propto}$

0.5

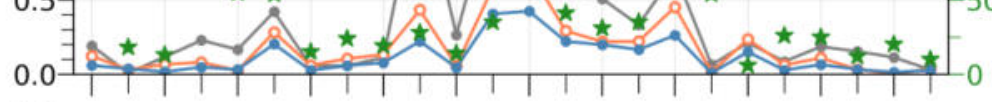

1.2 齐(e) $200-250 \mathrm{~km}$

1.0

0.6 刍

$0.4=$

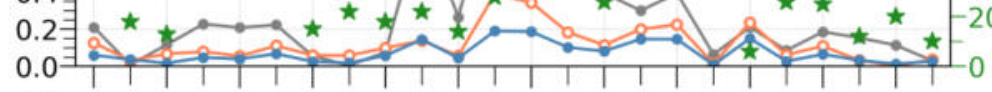

0.3 (f) $150-200 \mathrm{~km}$

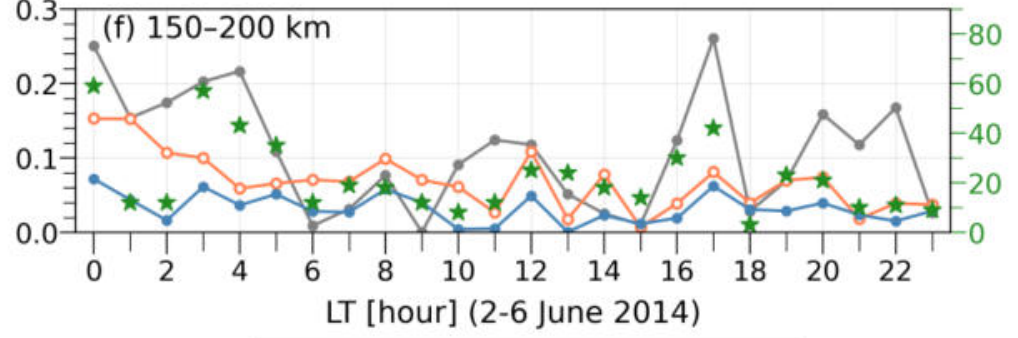

- TIE-GCM - Forecast $\bullet$ Analysis 


\section{Description of the next figure:}

The following figure show an example of the impact of assimilation on the forecasted electron density distribution compared to the 'normal' TIE-GCM run. These are multidimensional scatter plots revealing additional aspects of the compared electron density distributions.

The data points in orange indicate whether the particular COSMIC-Ne observation was assimilated in the next time step to produce the analysis state. In other words, the forecast shown here is an estimate of the electron density at the observation location with a lead time of one hour. The COSMIC observations that were discarded by the assimilation scheme in the update/analysis step are shown in gray. The forecast shown here is only influenced by COSMIC-Ne observations that were assimilated in previous time steps and not the particular observation that it is compared to in the current time step. The total number of rejected (assimilated) epochs in the distribution are noted as $R(A)$ in each scatter plot. 
$\mathrm{Pr}=$ Pearson's correlation coefficient between distributions specified in the abscissa and ordinate.

$\sigma C, \sigma T$, and $\sigma F$ give the standard deviations (in units of $10^{5} \mathrm{~cm}^{-3}$ ) of COSMIC, TIE-GCM, and forecast, respectively considering the entire population in the specified altitude region.

The solid blue line is a least squares polynomial fit of degree 1 considering all epochs, and the black dotted line represents the ideal data-model reference.

Figure also indicates the geographic latitude of the epochs in three categories: low ( $25^{\circ} \mathrm{S}-25^{\circ} \mathrm{N}$; triangle), middle $\left(25^{\circ}-60^{\circ} \mathrm{N} / \mathrm{S}\right.$; cross), and high ( $60^{\circ}-90^{\circ}$ N/S; dot).
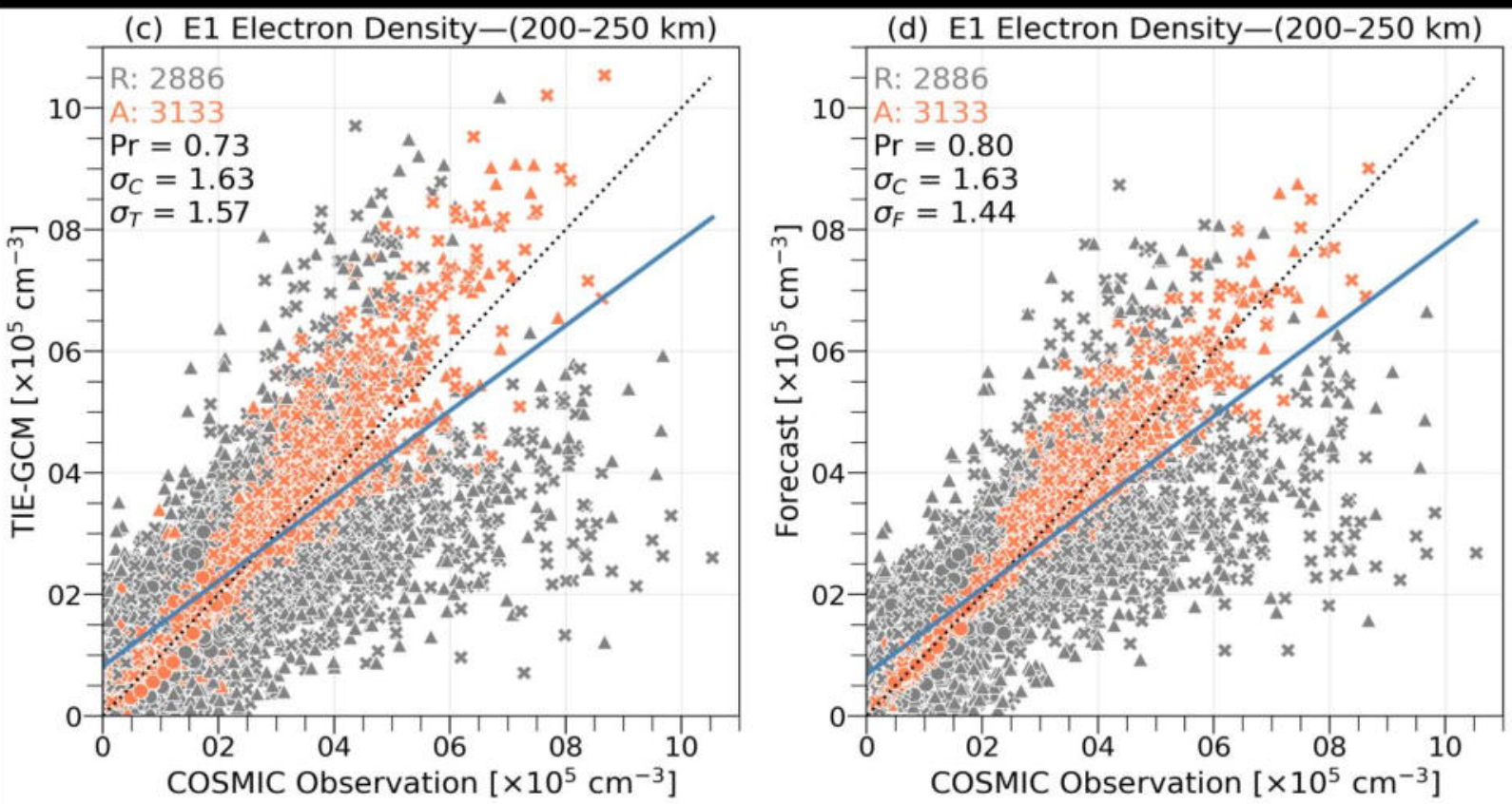

(e) E1 Electron Density-(150-200 km)
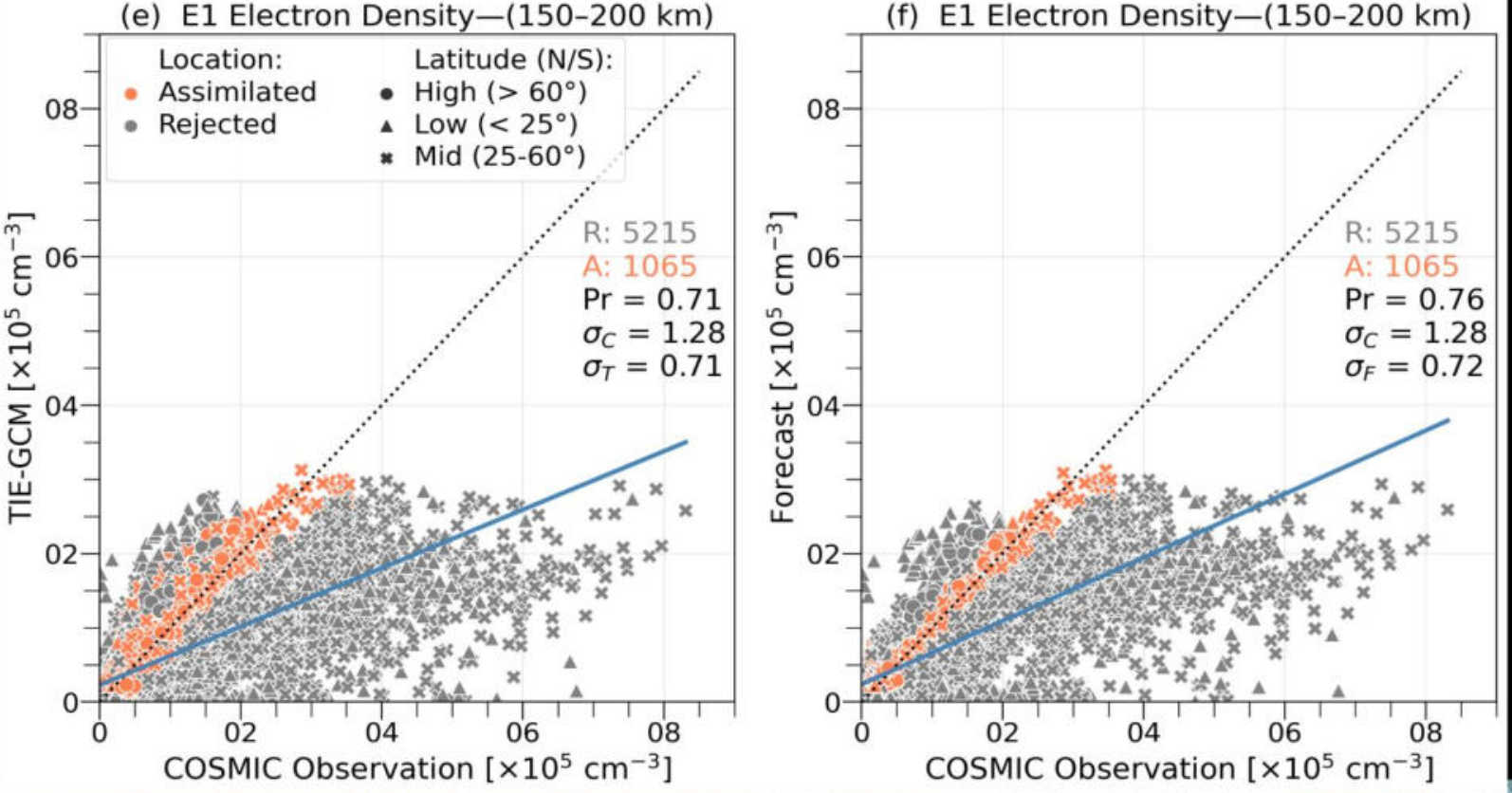

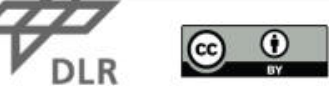



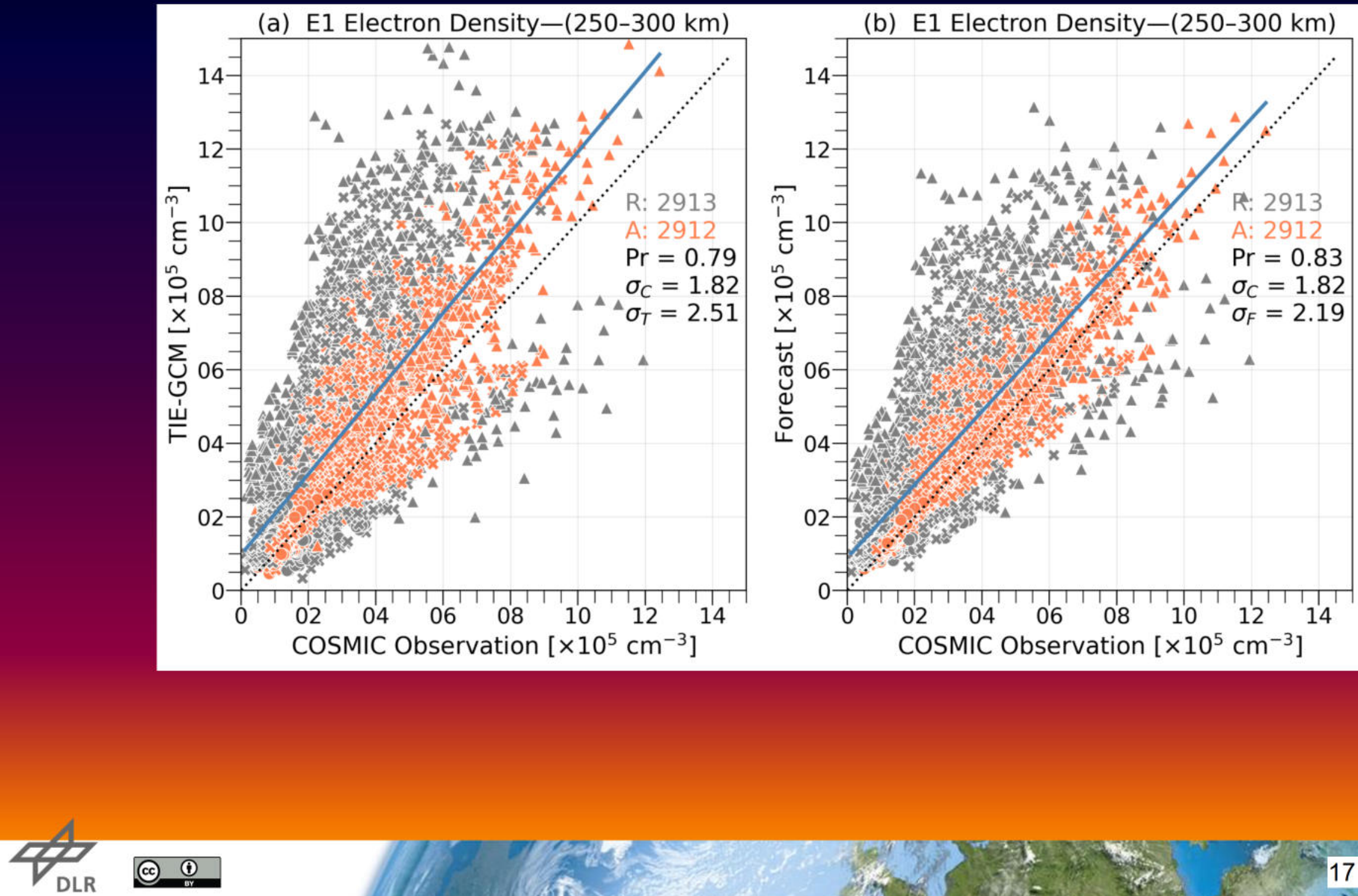
Same as above except for E2.
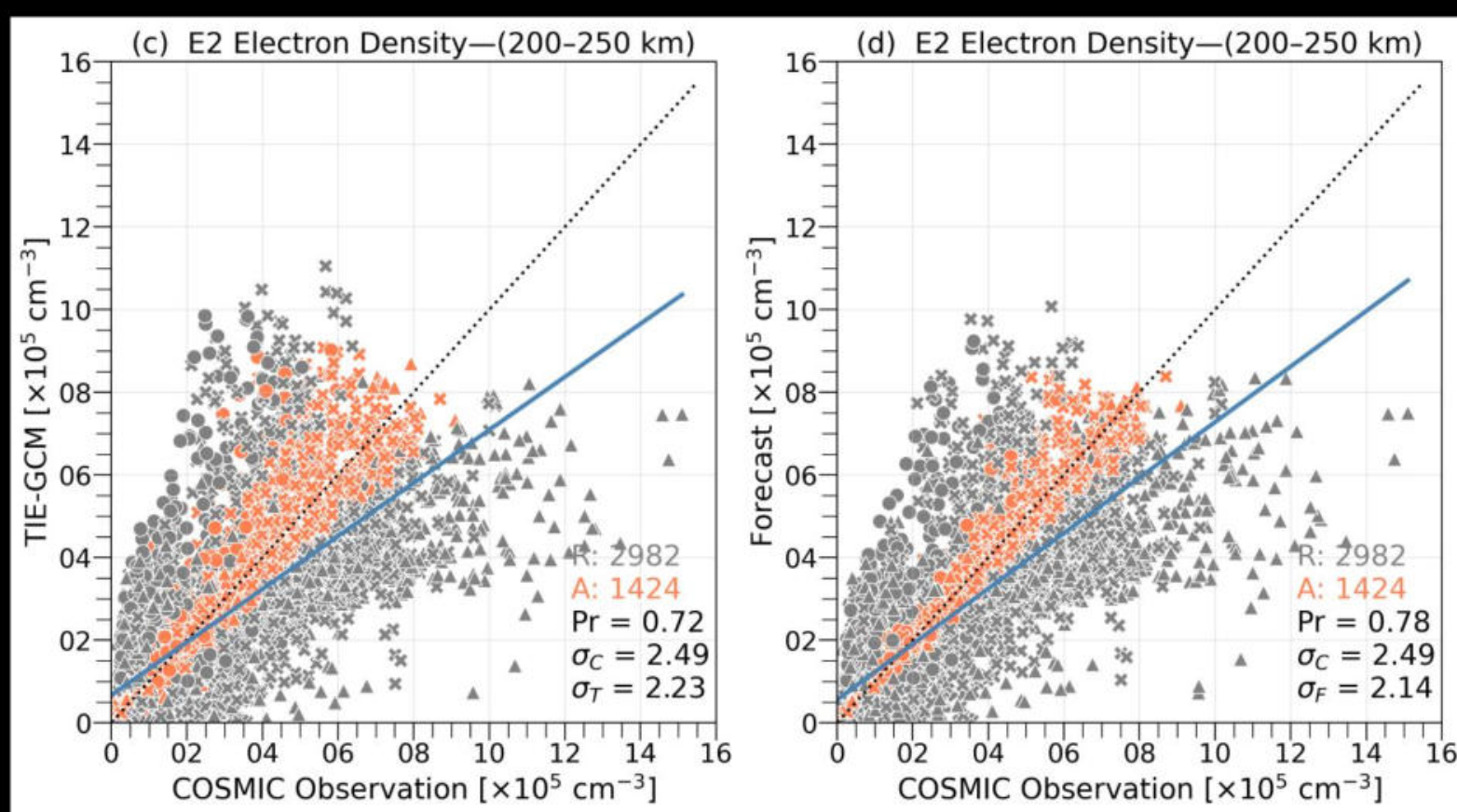

(e) E2 Electron Density-(150-200 km)

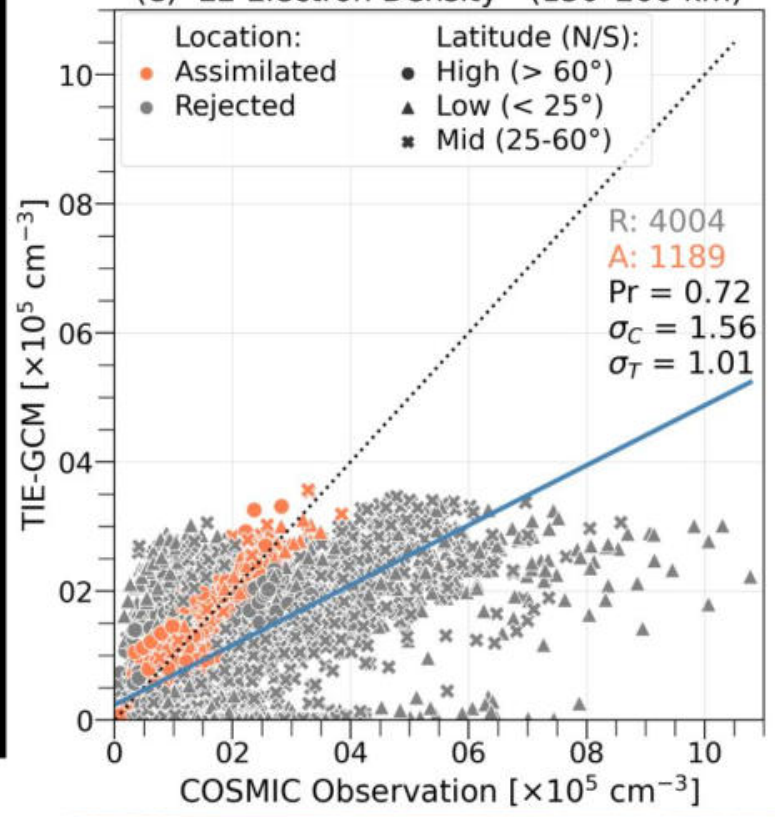

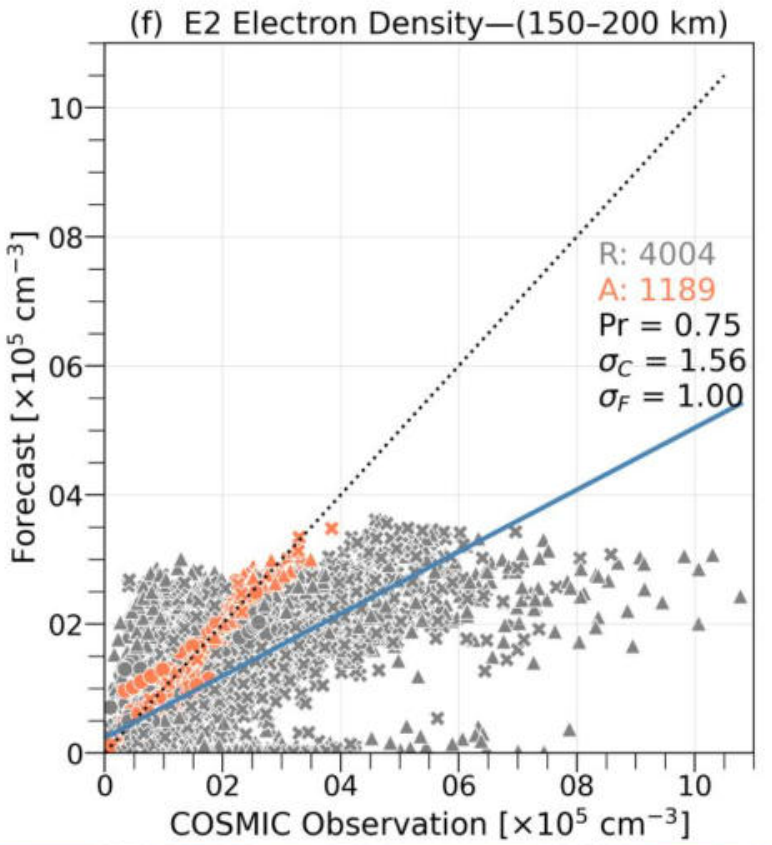



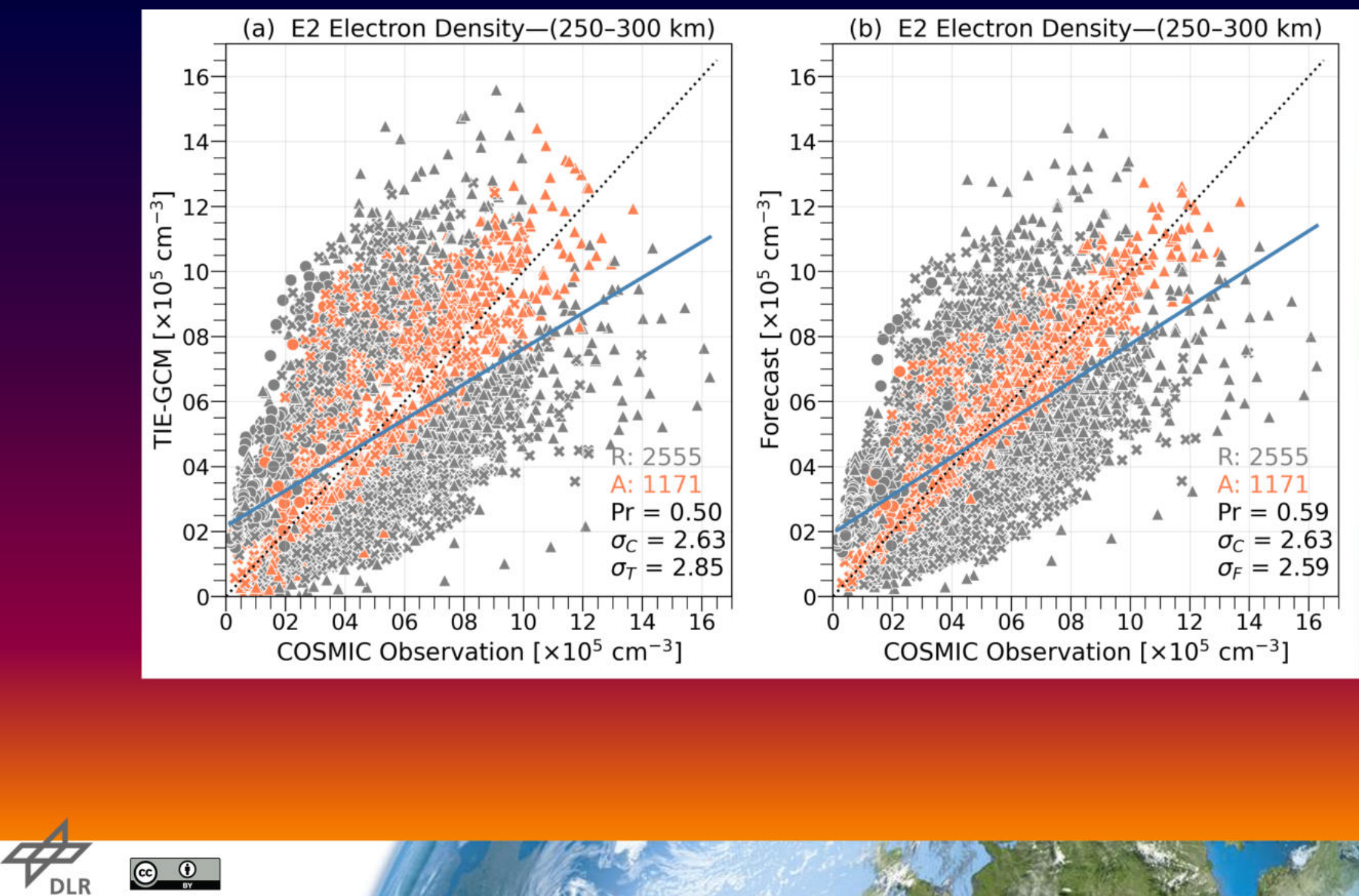
The following figure summarizes the assimilation results of both E1 and E2 relative to COSMIC observations. 

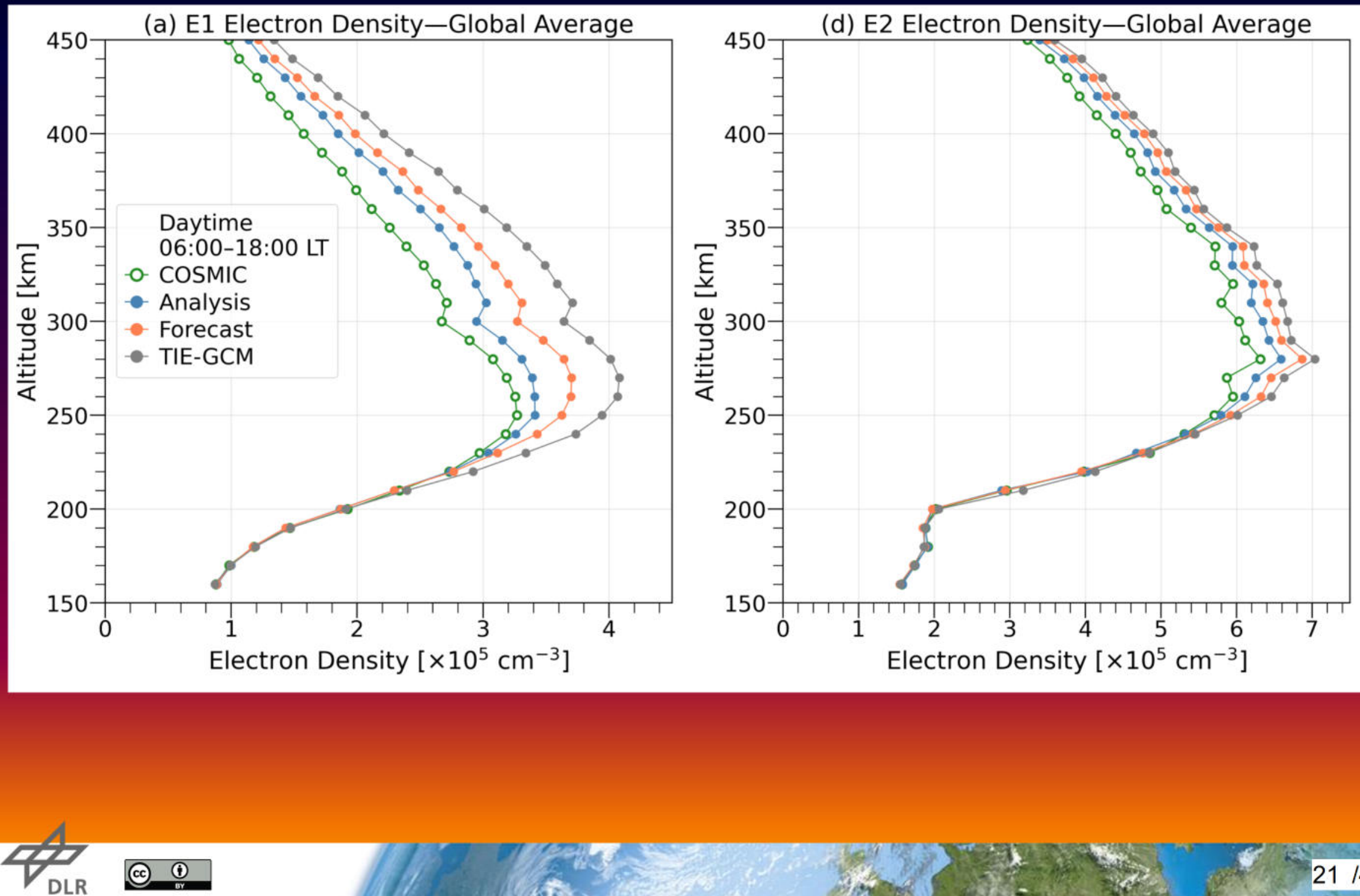
Daytime

$\sigma C, \sigma T,, \sigma A$, and $\sigma F$ give the standard deviations (in units of $10^{5} \mathrm{~cm}^{-3}$ ) of COSMIC, TIE-GCM, analysis, and forecast, respectively considering the entire population in the specified altitude region.

The solid black line is a least squares polynomial fit of degree 1 considering all epochs, and the black dotted line represents the ideal data-model reference.

Here the data are binned by the resolution of the input COSMIC data (i.e. $10 \mathrm{~km}$ ). (b) E1 Average by Altitude-TIE-GCM

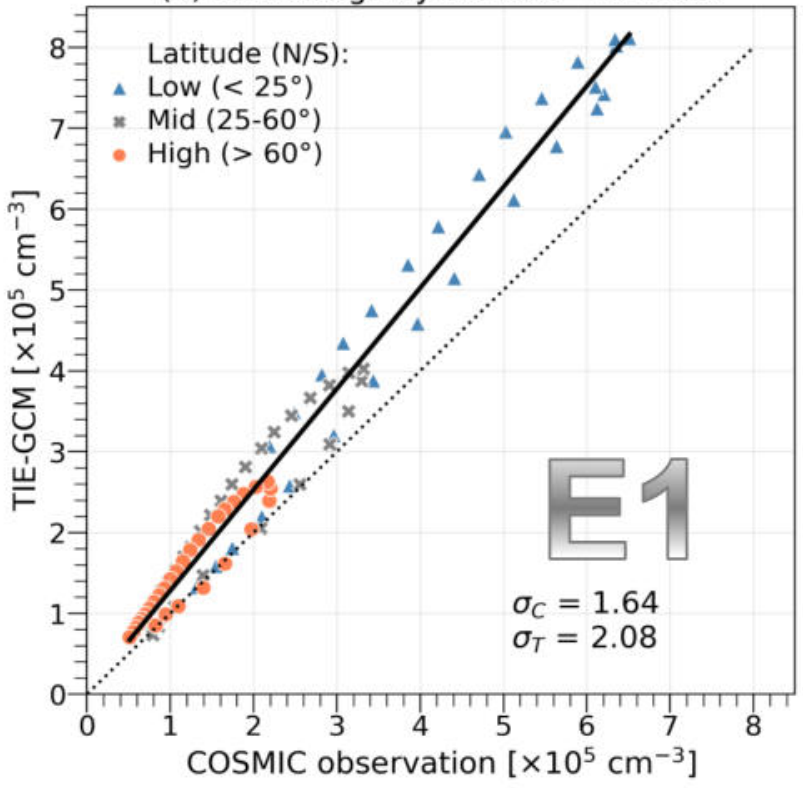

(c) E1 Average by Altitude-Assimilation

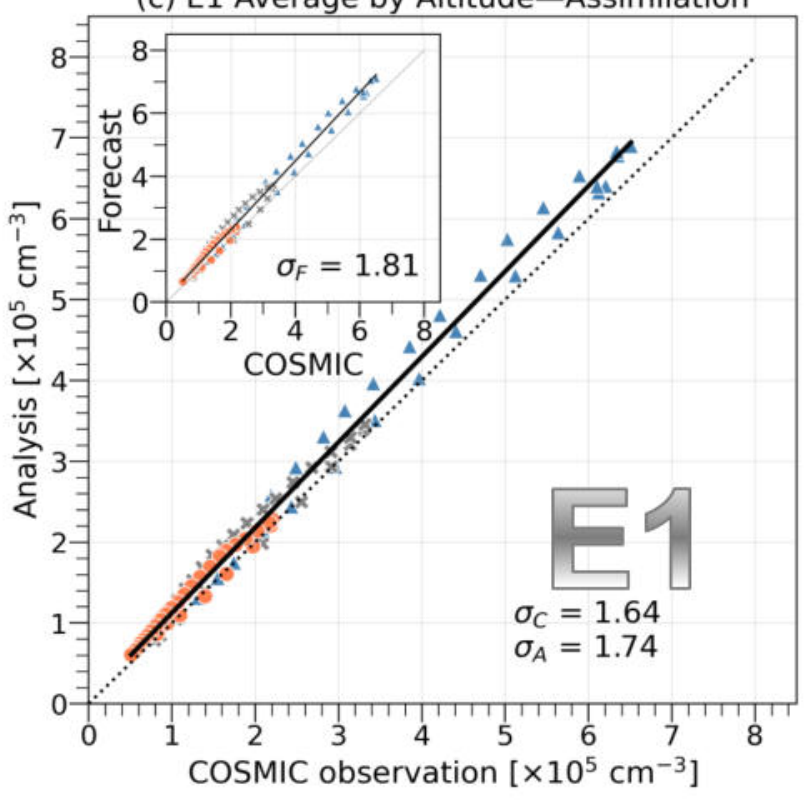

(e) E2 Average by Altitude-TIE-GCM

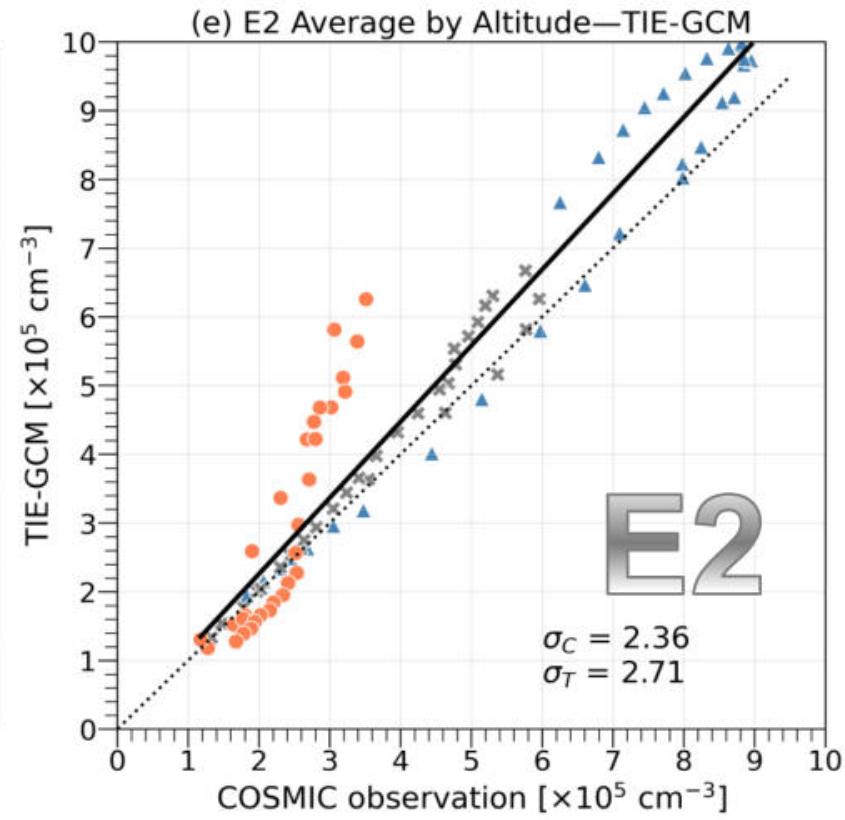

(f) E2 Average by Altitude-Assimilation

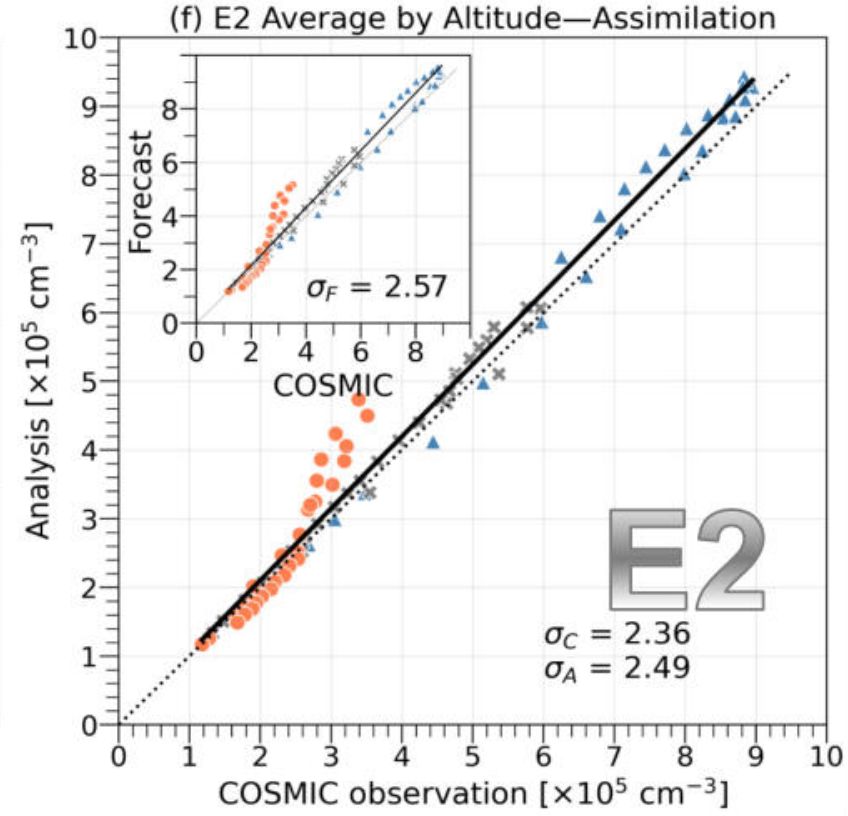


The following figure is similar to the previous figure except for night-time electron density profile. 

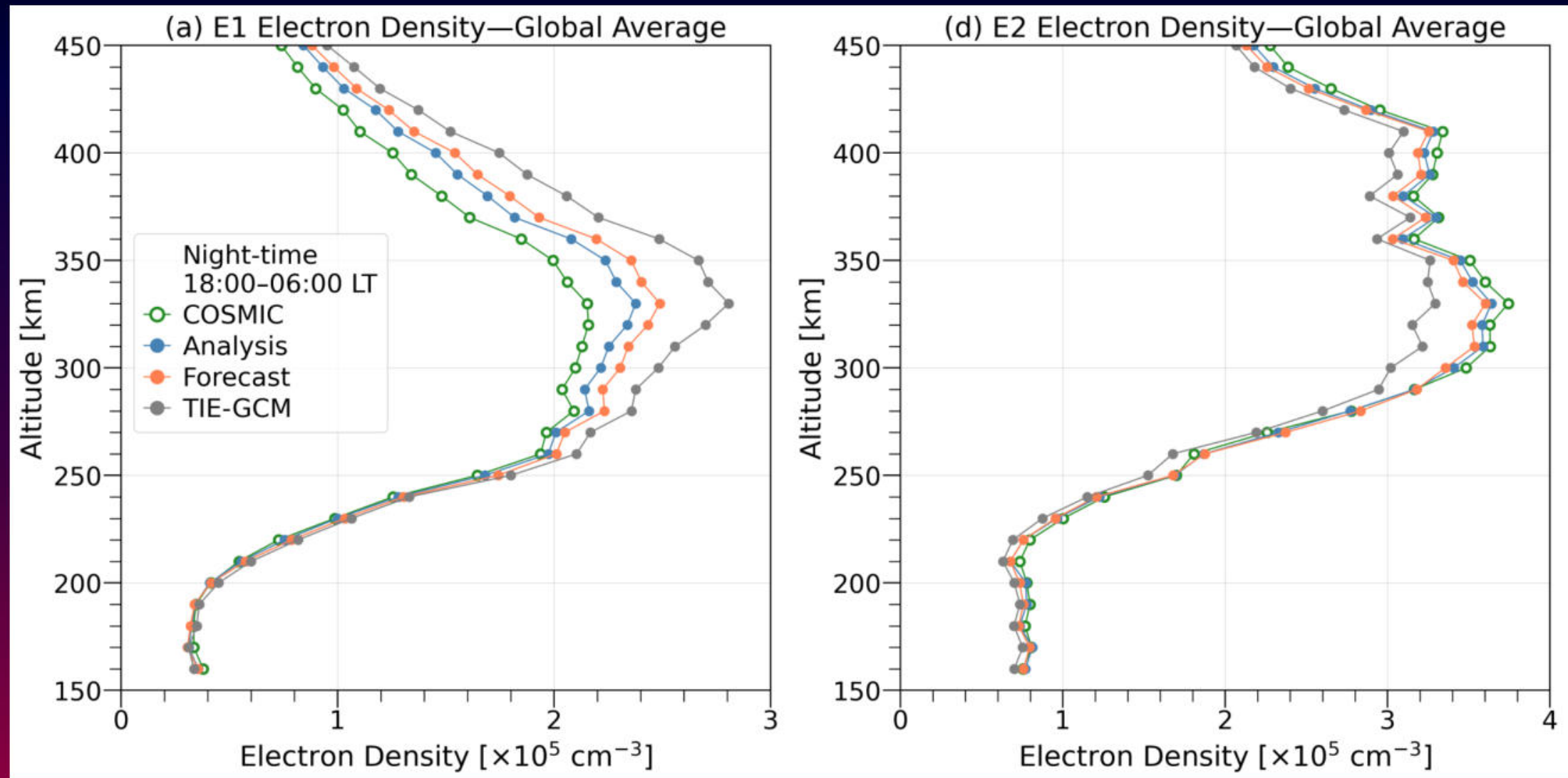
Night-time

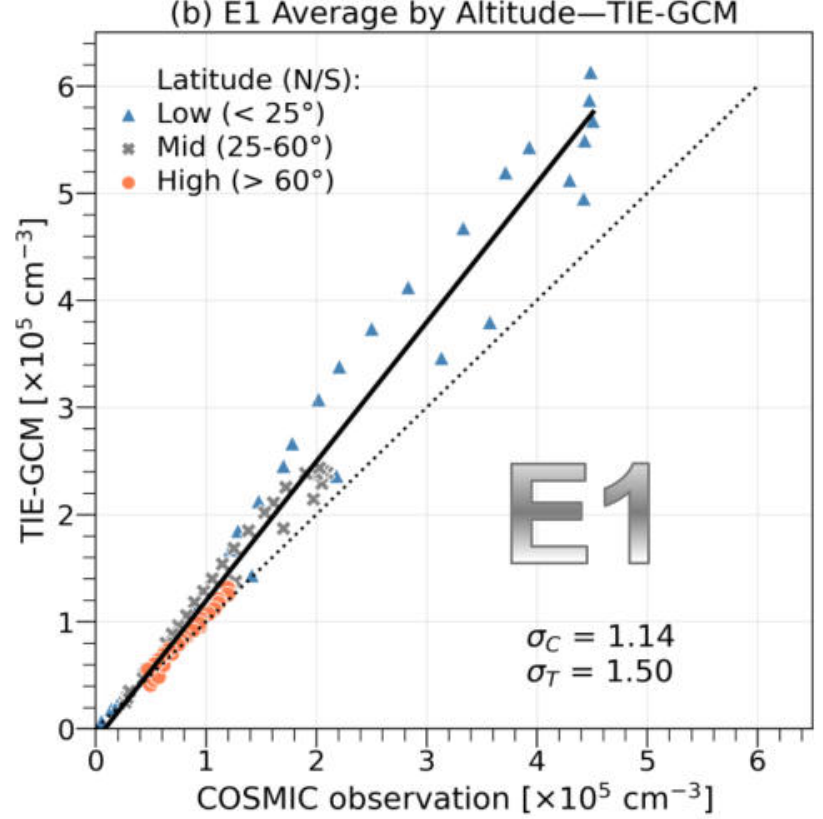

(c) E1 Average by Altitude-Assimilation

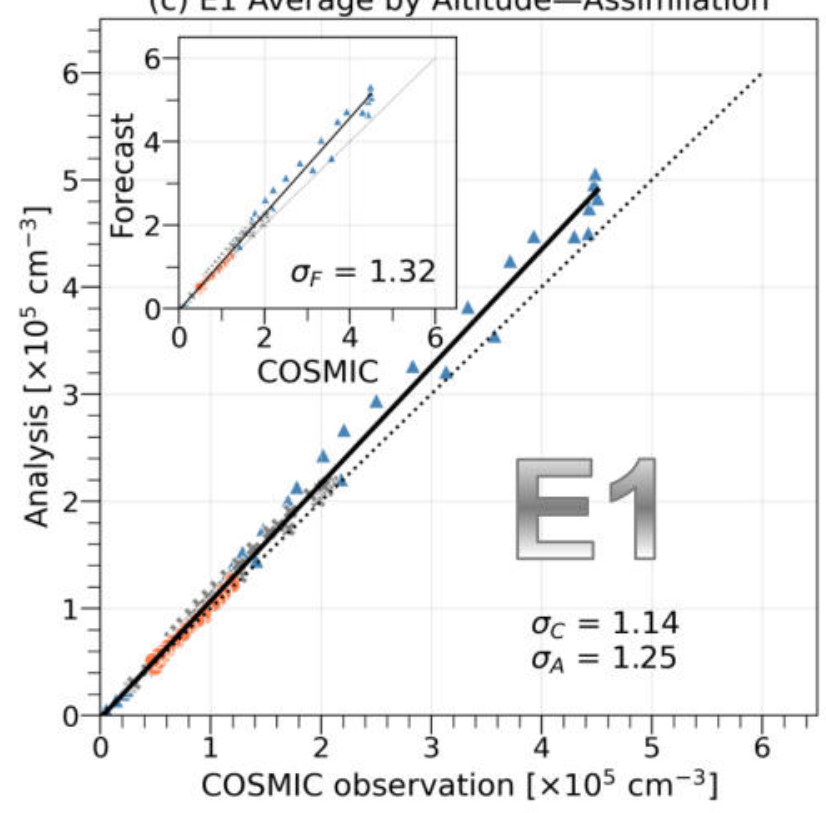

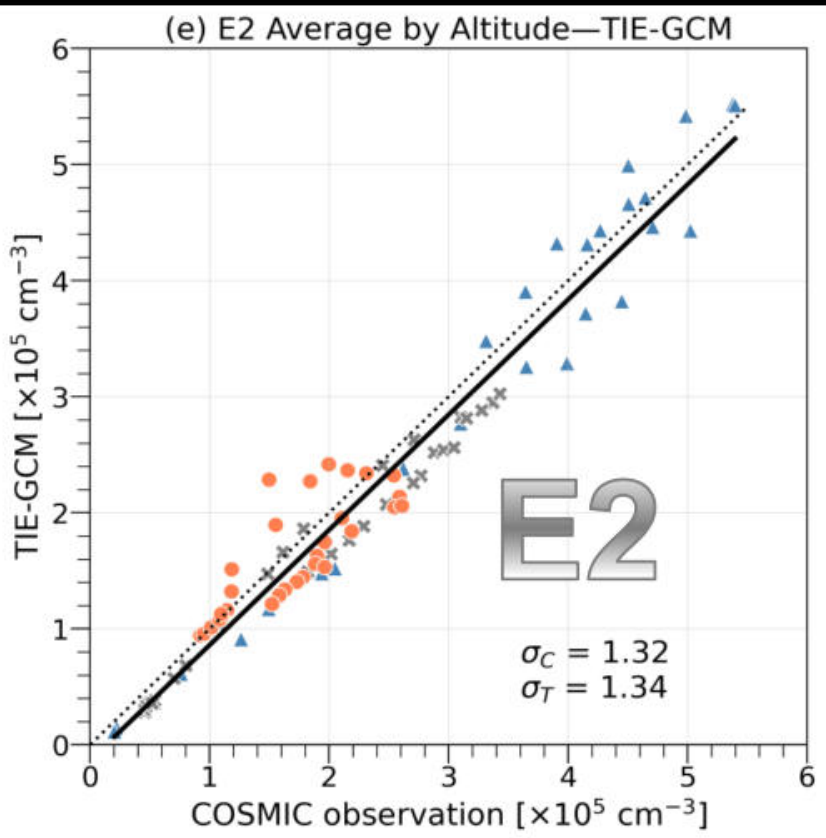

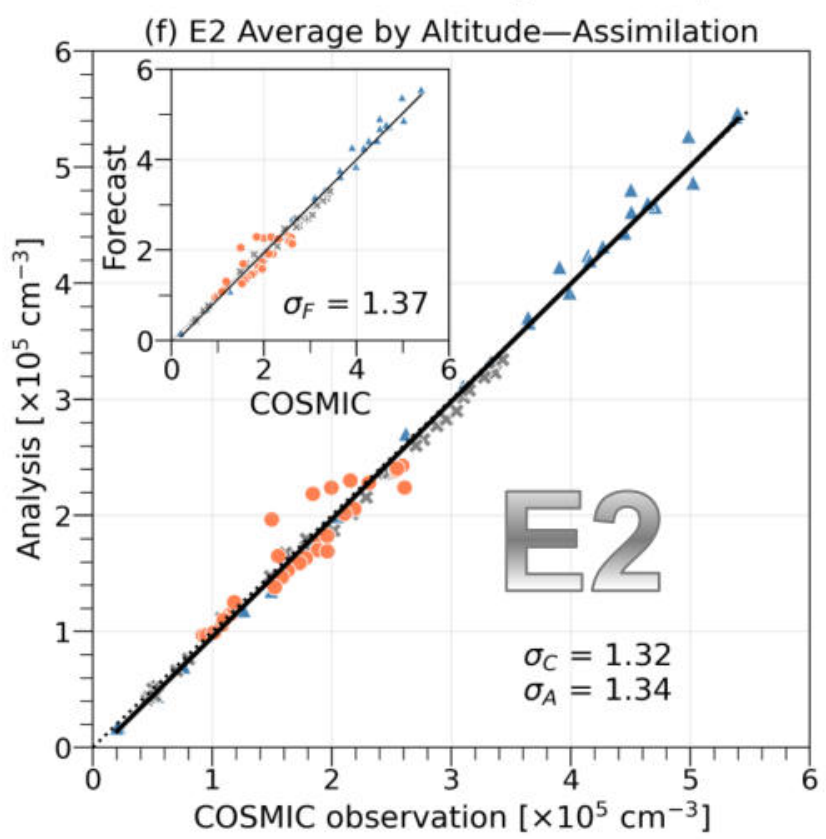

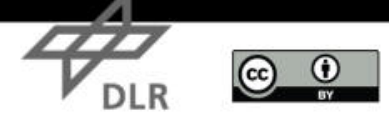

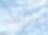




\section{Validation of Forecasts Against Independent Data}

\section{E1}

E1 compared to GRACE electron density profile retrieved through $\mathrm{RO}$.
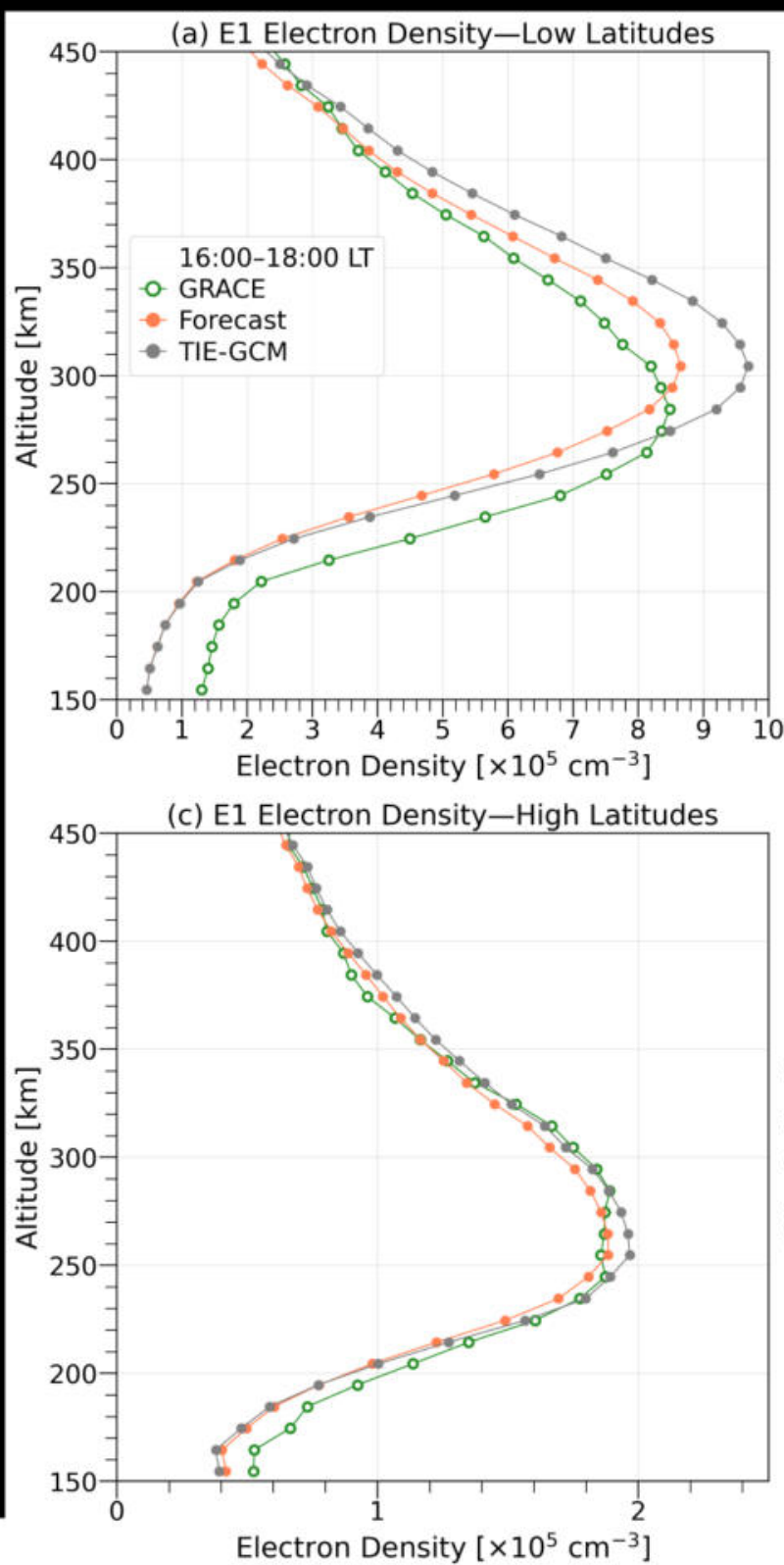

Electron Density $\left[\times 10^{5} \mathrm{~cm}^{-3}\right]$
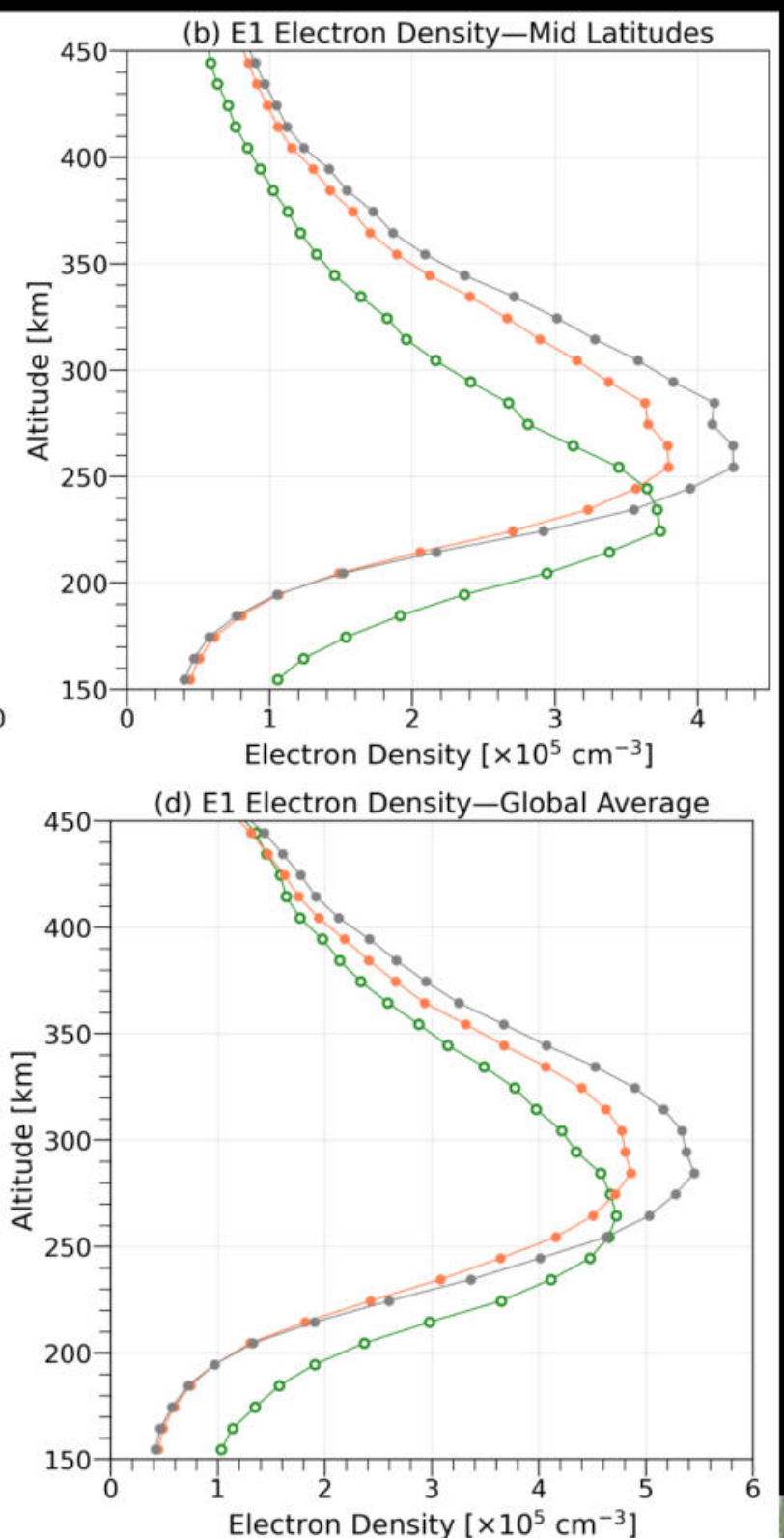

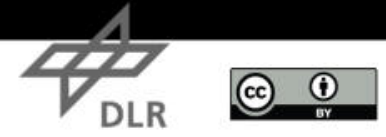

Electron Density $\left[\times 10^{5} \mathrm{~cm}^{-3}\right]$ 


\section{E1}
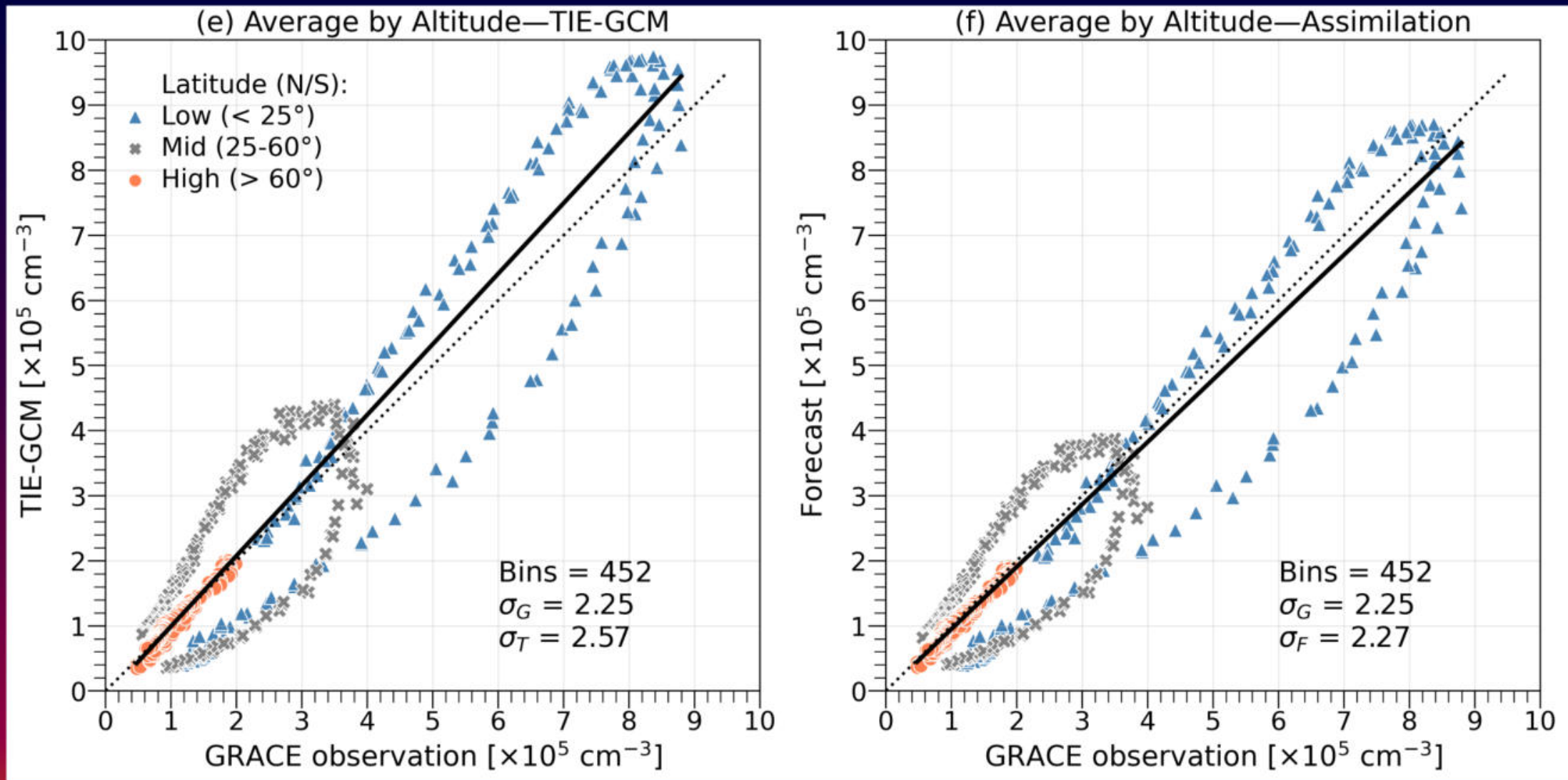


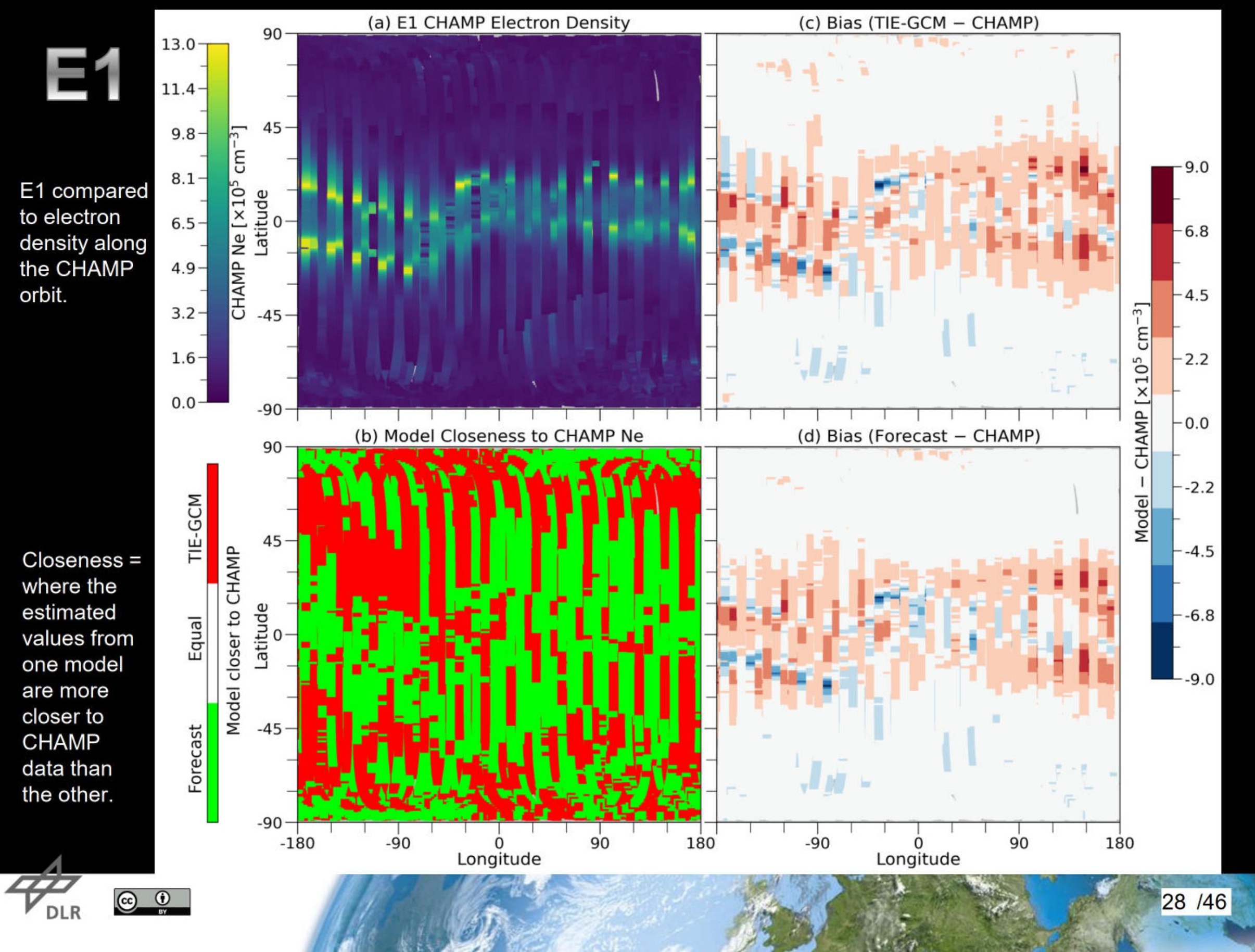


(a) E1 CHAMP Ne-Descending

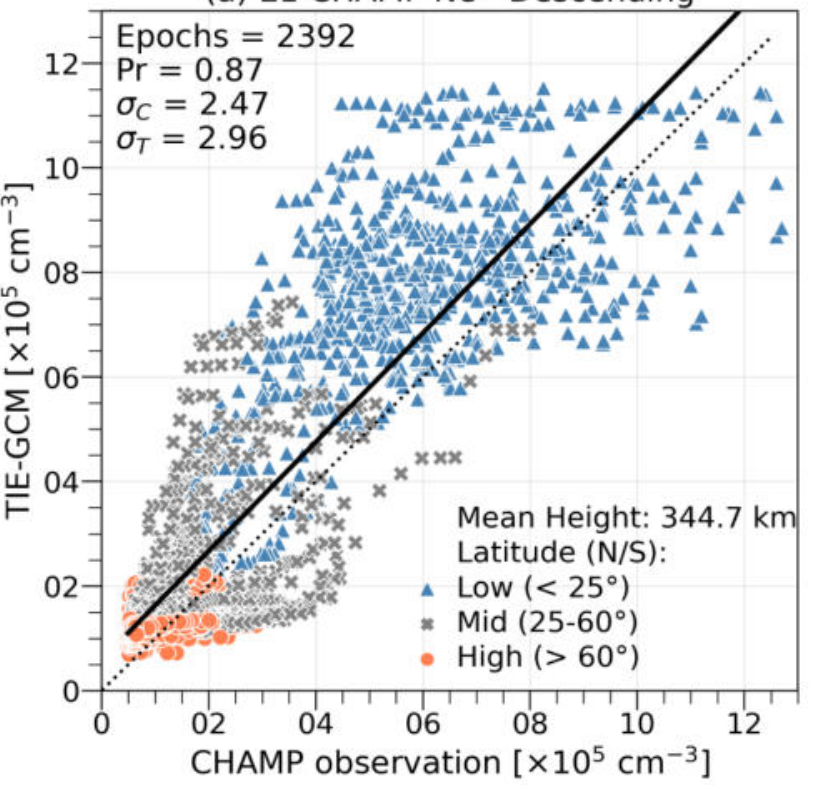

(c) E1 CHAMP Ne-Ascending

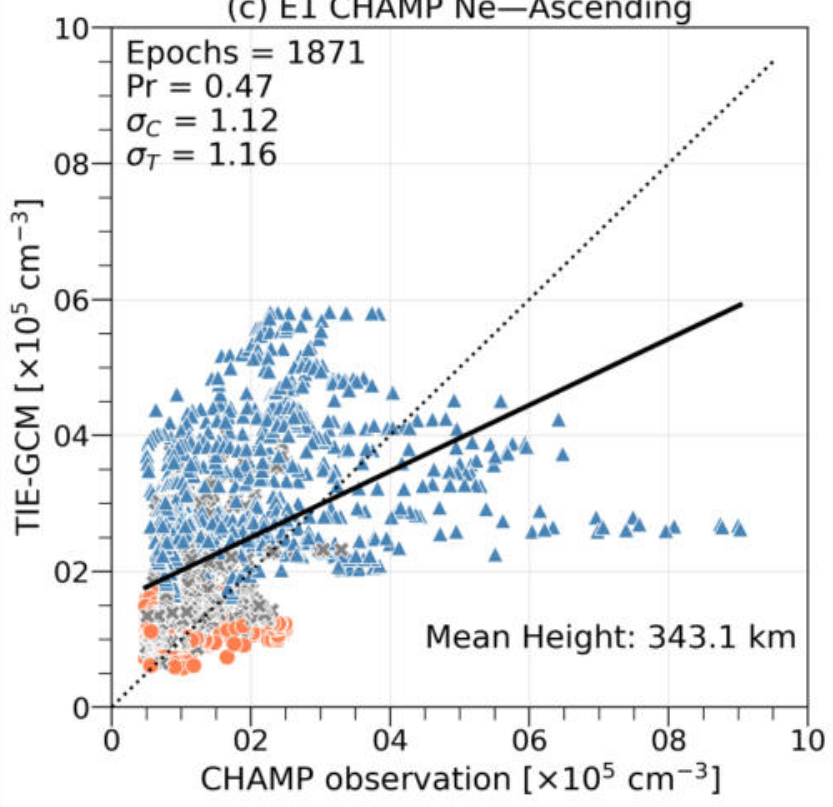

(b) E1 CHAMP Ne-Descending

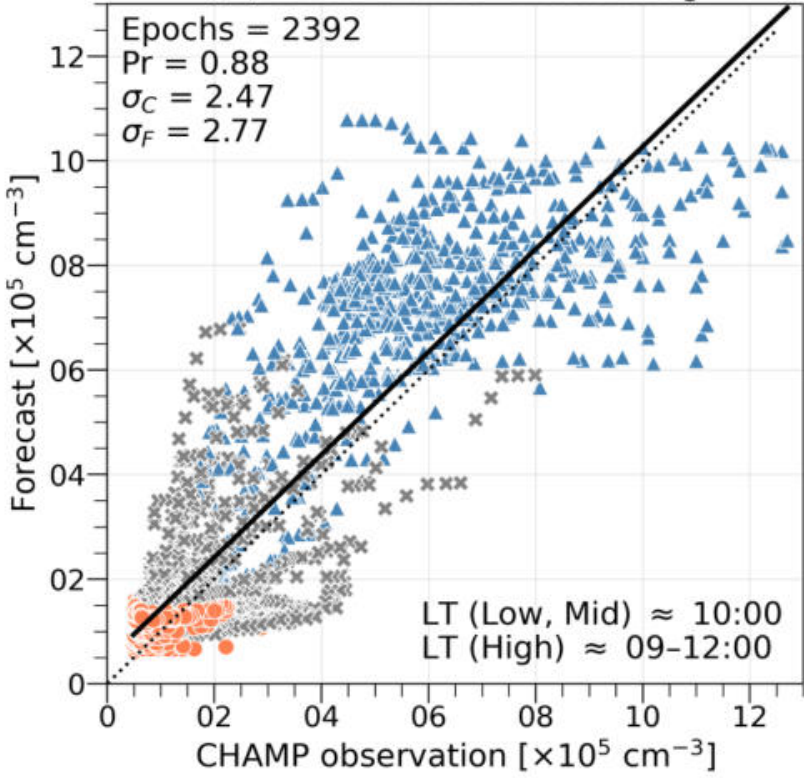

(d) E1 CHAMP Ne-Ascending

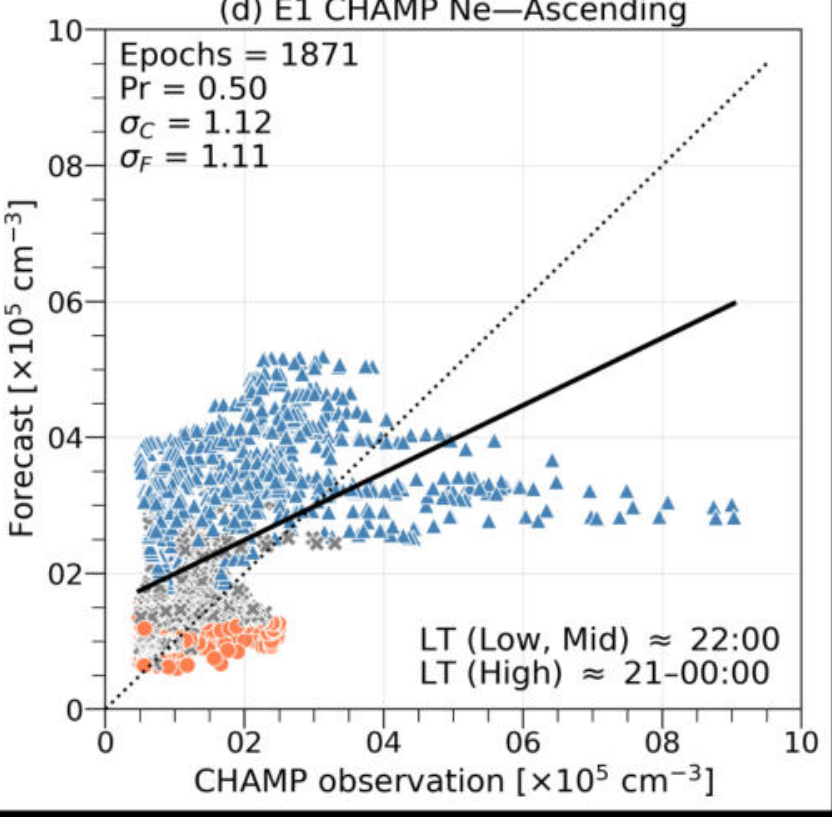

E1 compared to electron density along the CHAMP orbit's descending and ascending segments

.
s

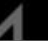



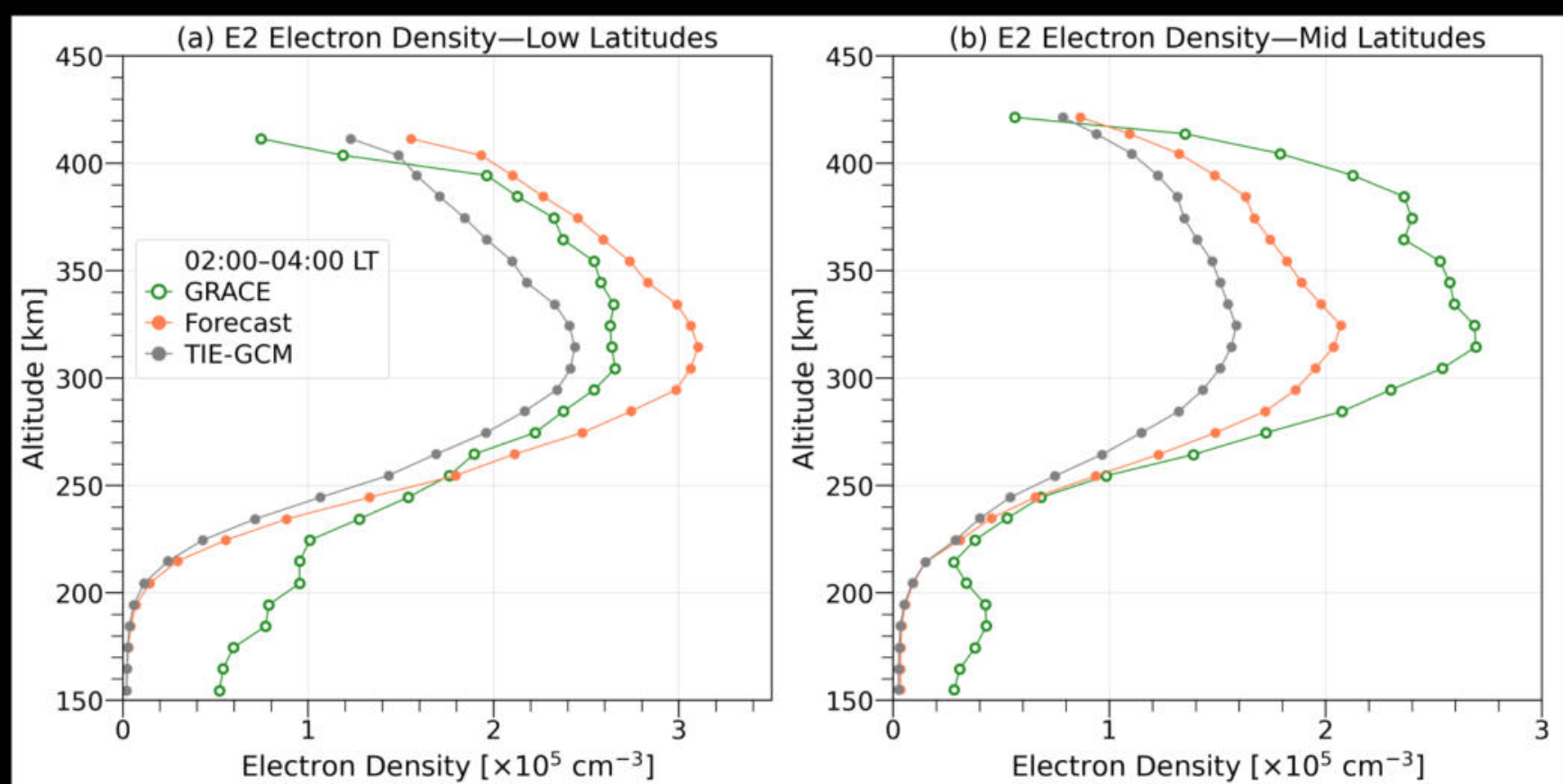

\section{E2 compared to GRACE electron density profile retrieved through RO.}

Electron Density $\left[\times 10^{5} \mathrm{~cm}^{-3}\right]$

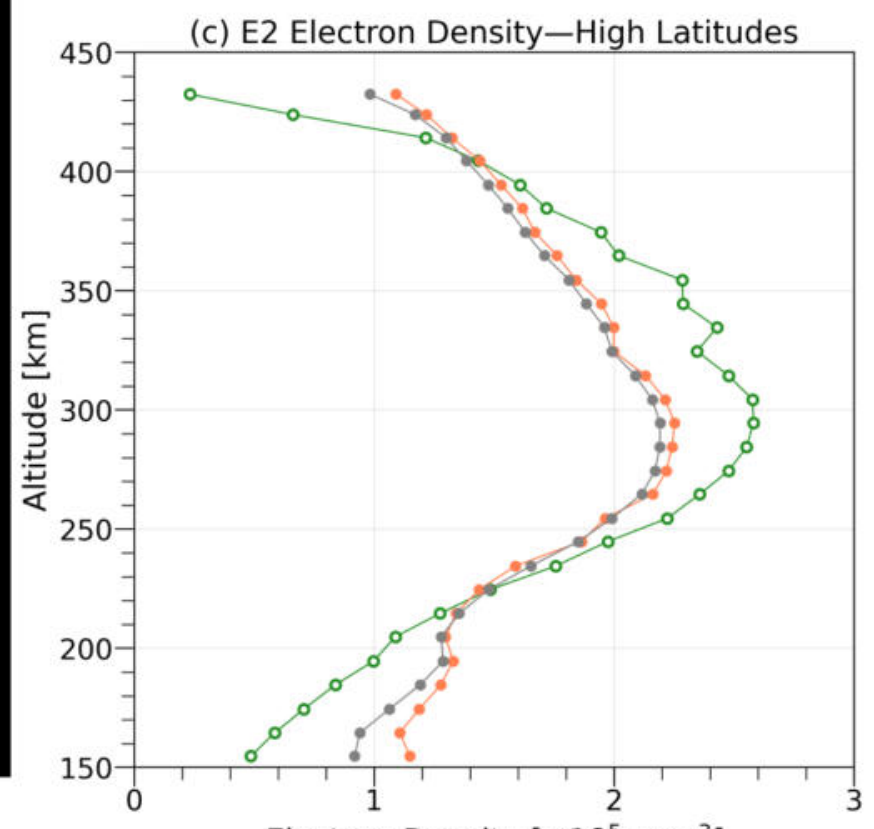

Electron Density $\left[\times 10^{5} \mathrm{~cm}^{-3}\right]$ (d) E2 Electron Density-Global Average

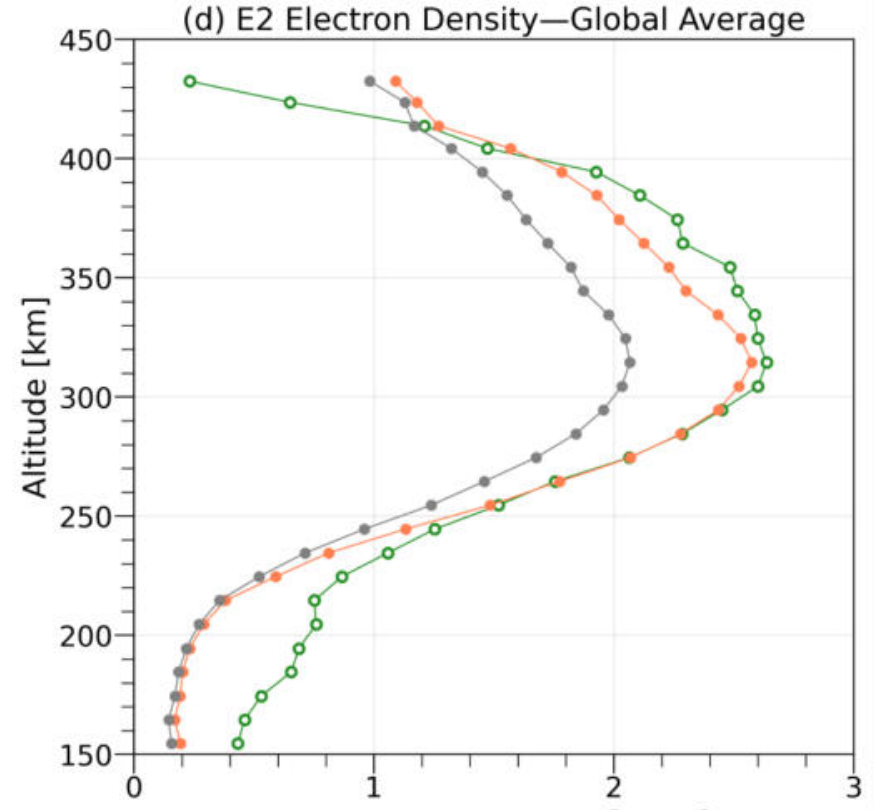

Electron Density $\left[\times 10^{5} \mathrm{~cm}^{-3}\right]$ 


\section{E2}

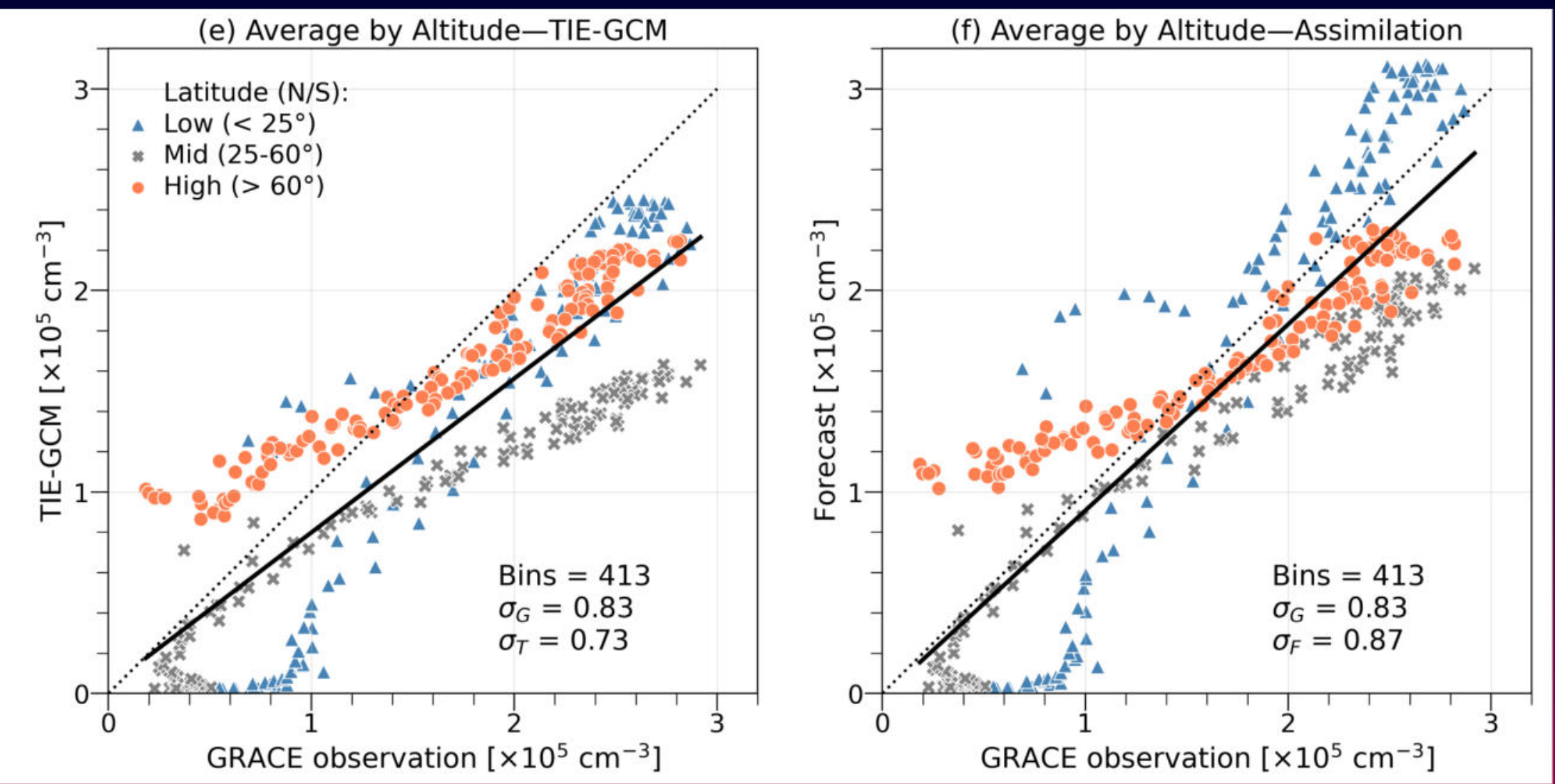




\section{Impact of the Assimilation on the Thermosphere}

The following figures show longitude-latitude maps of neutral mass density $(\rho)$ at a few selected altitude levels and UTs. The left column shows the estimated $\rho$ from TIE-GCM, forecast, and analysis runs. Their differences are shown in the right column. The cyan dots indicate the location of COSMIC-Ne data that were assimilated since 2.5 hours prior to the time indicated in the snapshot. 
E1 Neutral Mass Density-2008-03-04 01 UT, 250 km

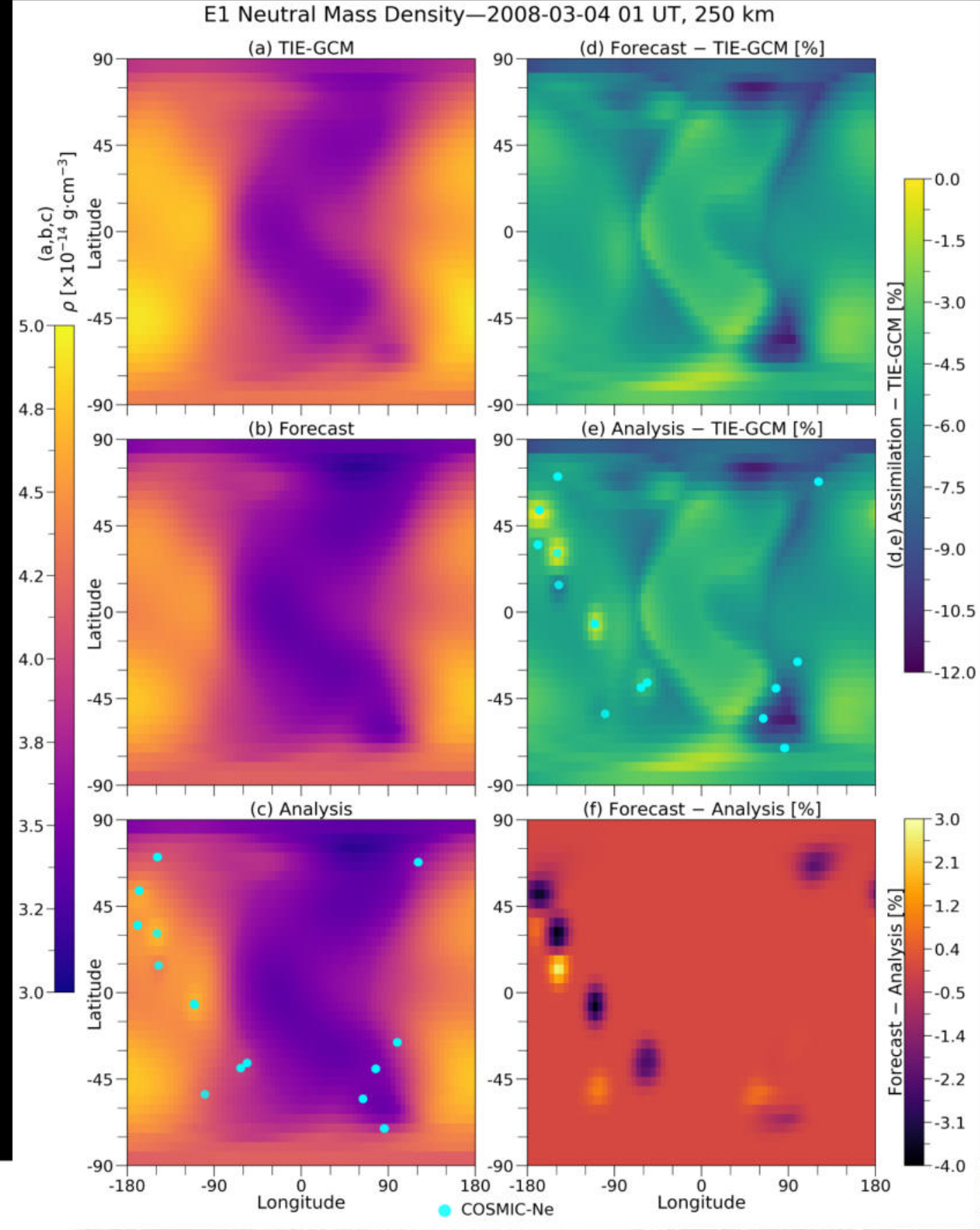


E1 Neutral Mass Density-2008-03-04 01 UT, 350 km

(a) TIE-GCM

(d) Forecast - TIE-GCM [\%]
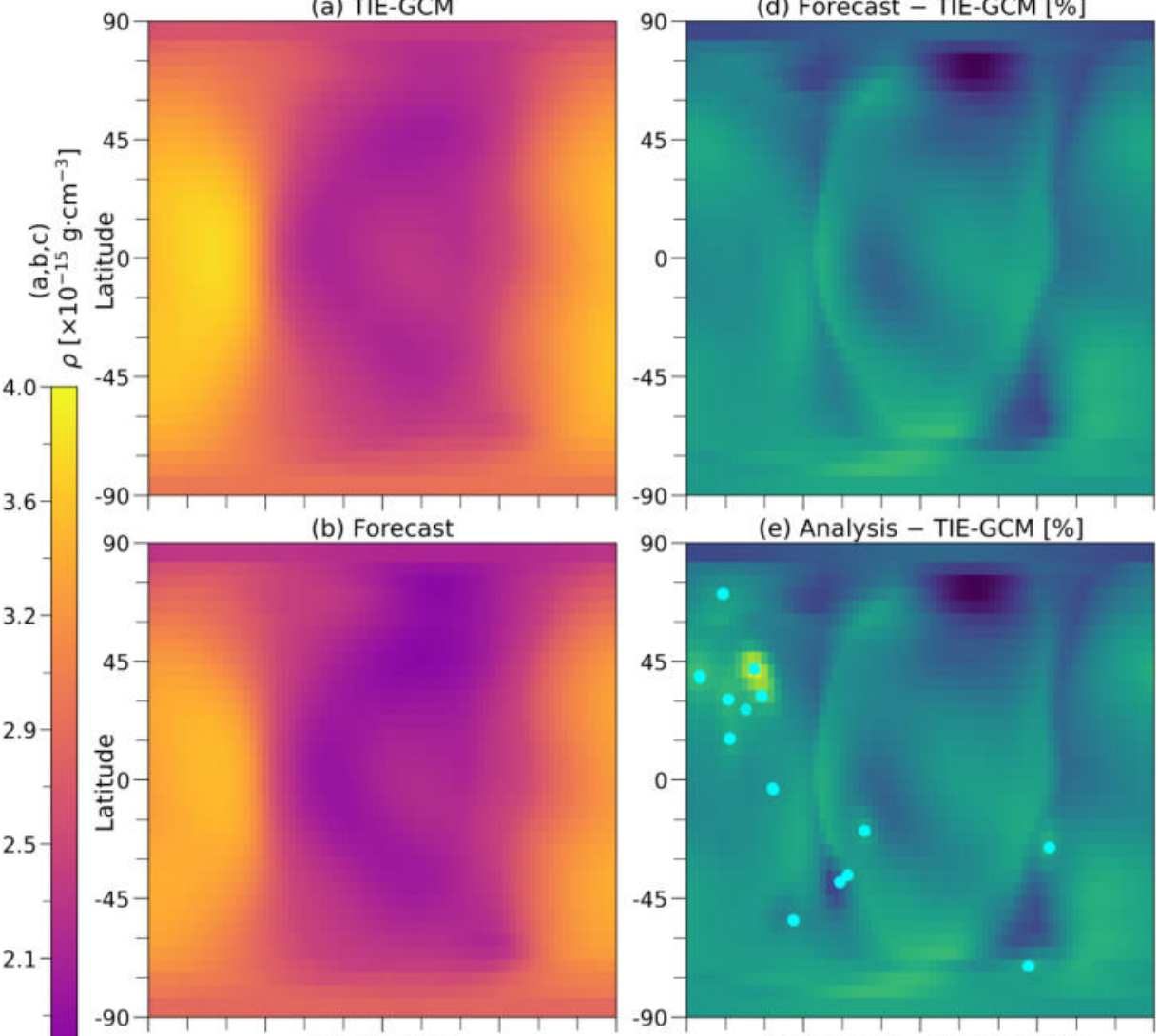

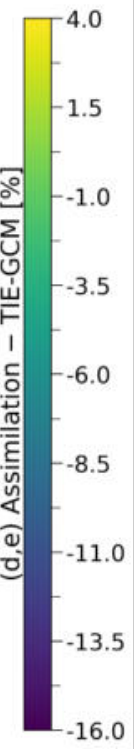

(c) Analysis

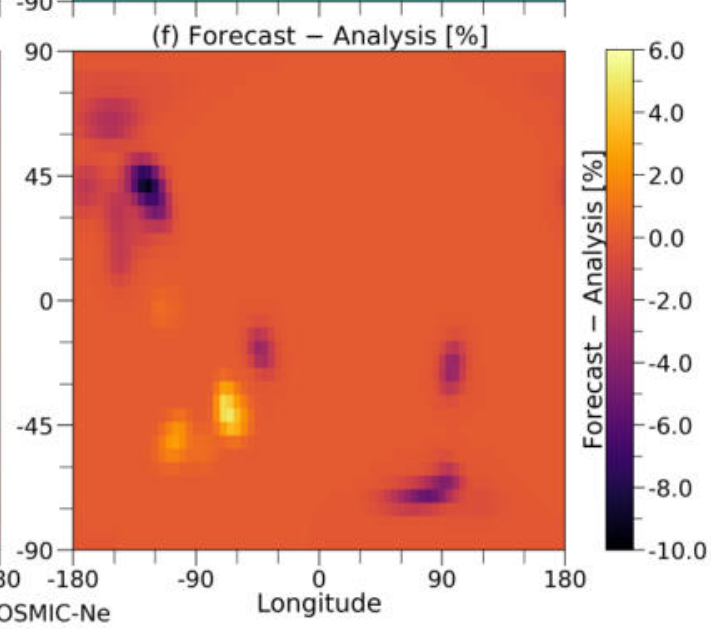

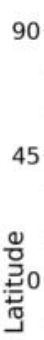

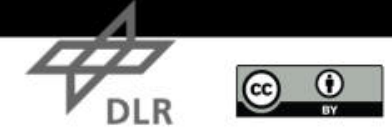

$1.4-1.8$
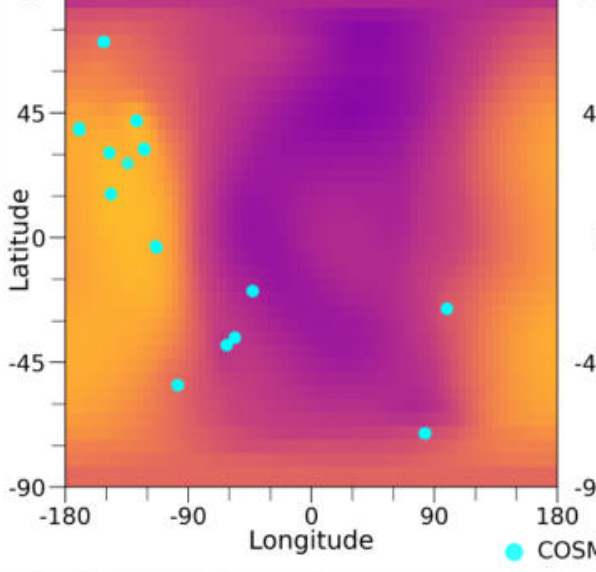

90
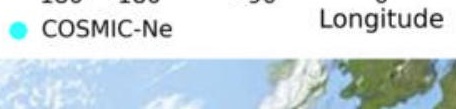

genthors 
E1 Neutral Mass Density-2008-03-04 01 UT, 450 km

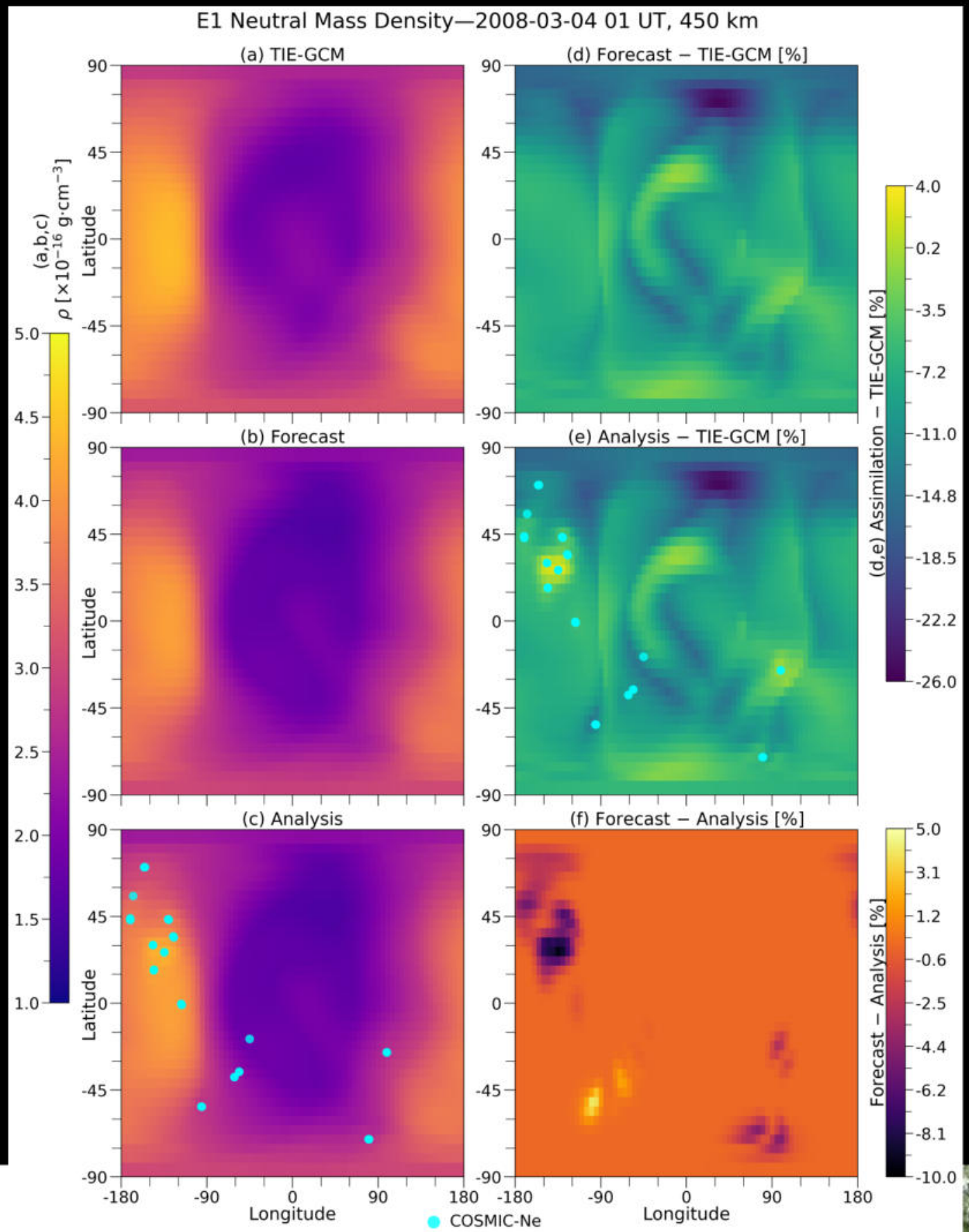


E1 Neutral Mass Density-2008-03-06 09 UT, 350 km

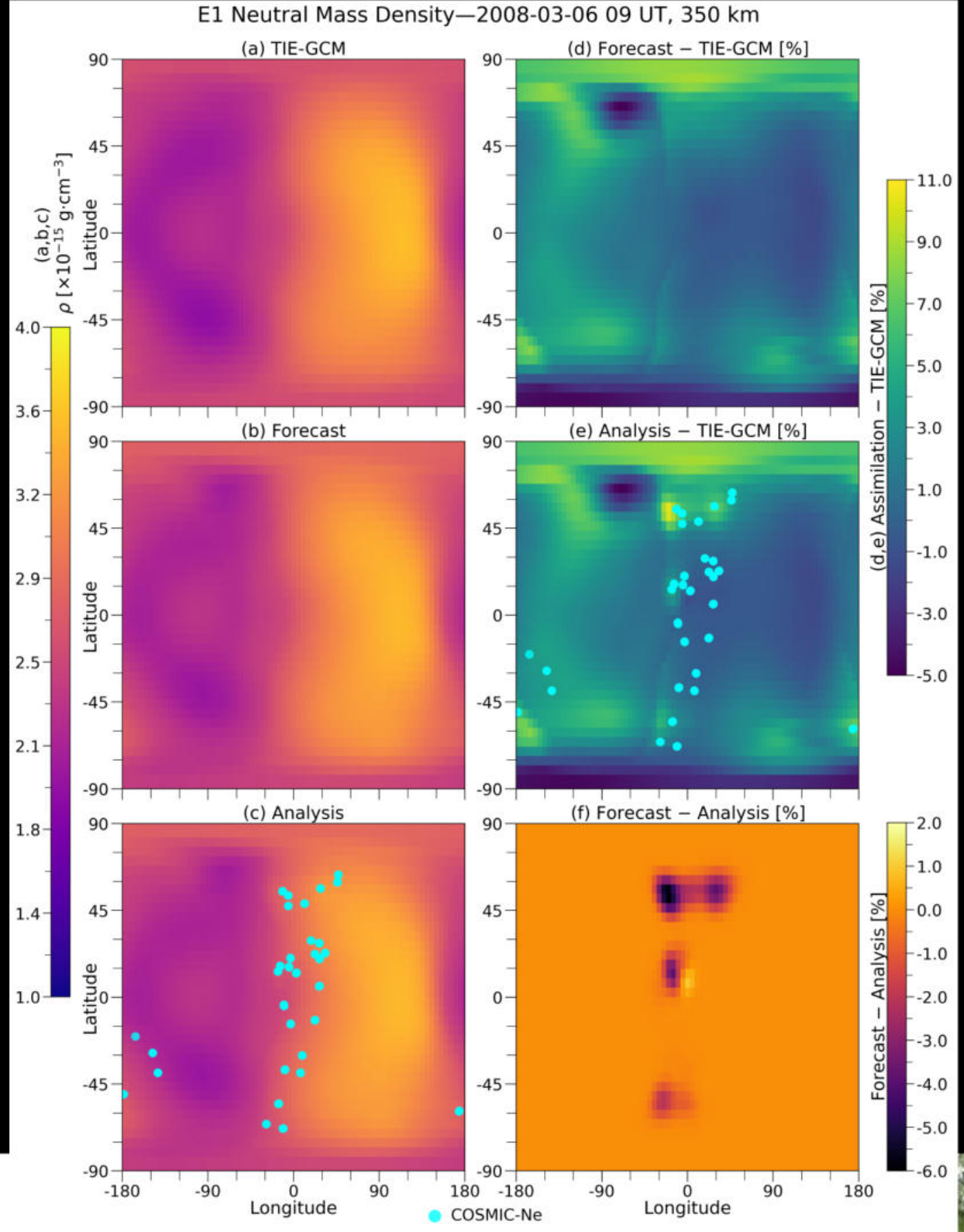


E1 Neutral Mass Density-2008-03-06 09 UT, 450 km
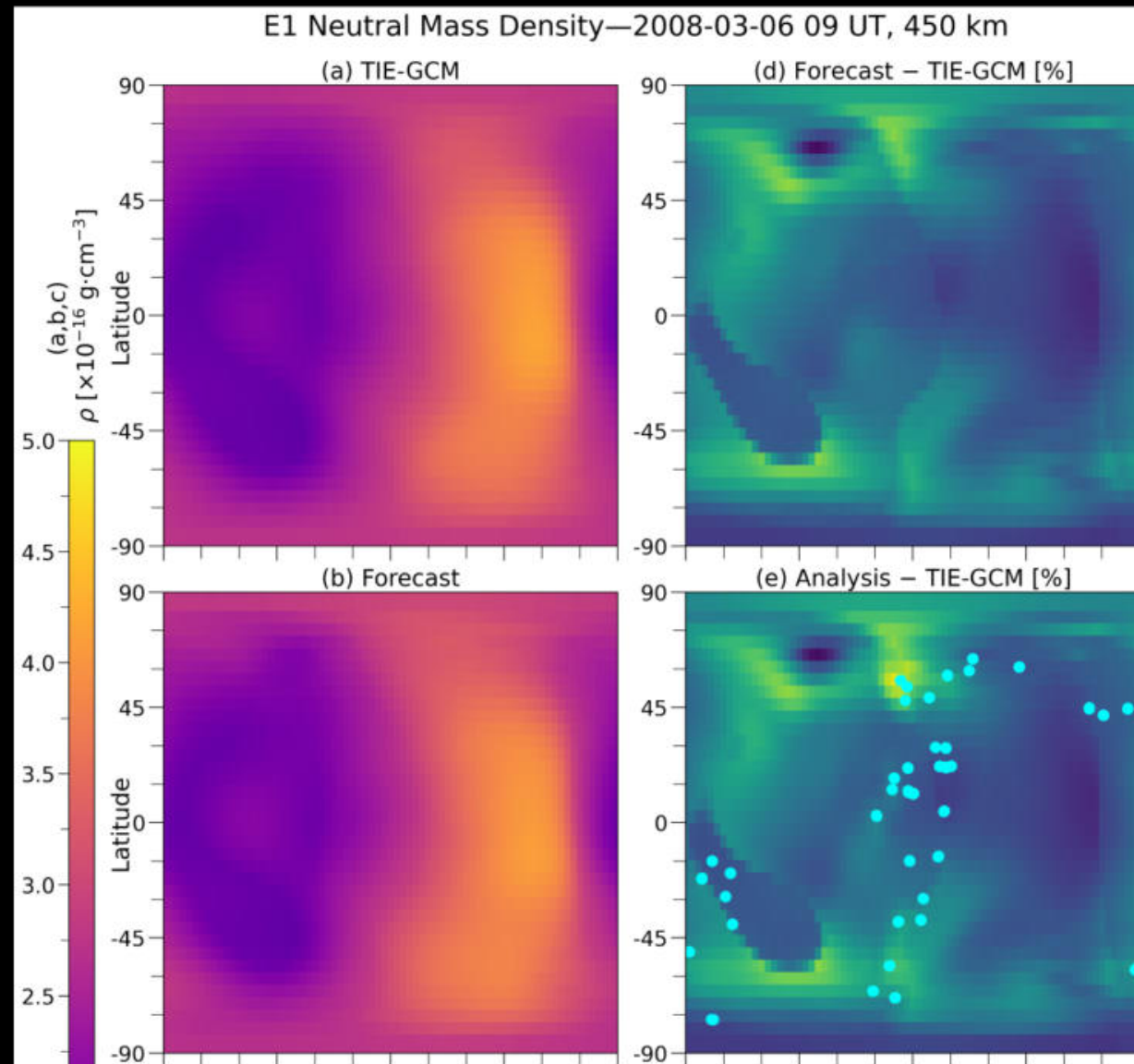

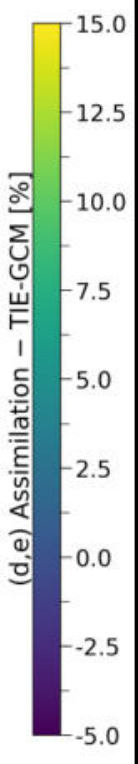

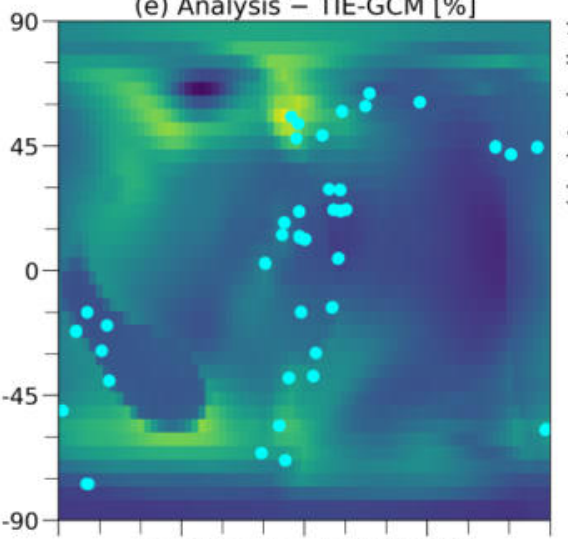

(c) Analysis

(f) Forecast - Analysis [\%]

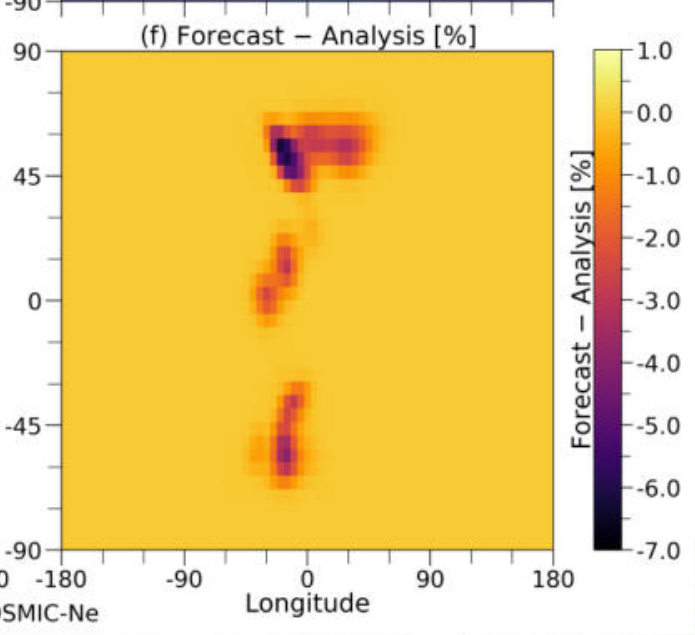

${ }_{1.0}^{2.0}$



$8.0 .8 \div 45$

80

$\bullet$

$\because$

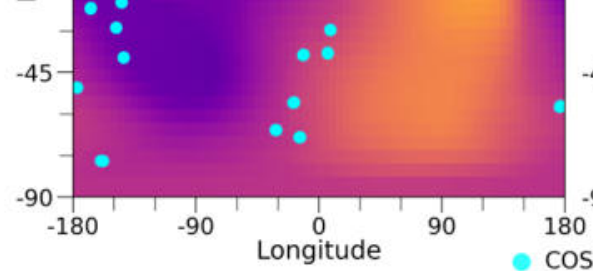

COSMIC-Ne

\section{Fis}


Same as above except for E2. 
E2 Neutral Mass Density-2014-06-02 01 UT, 250 km

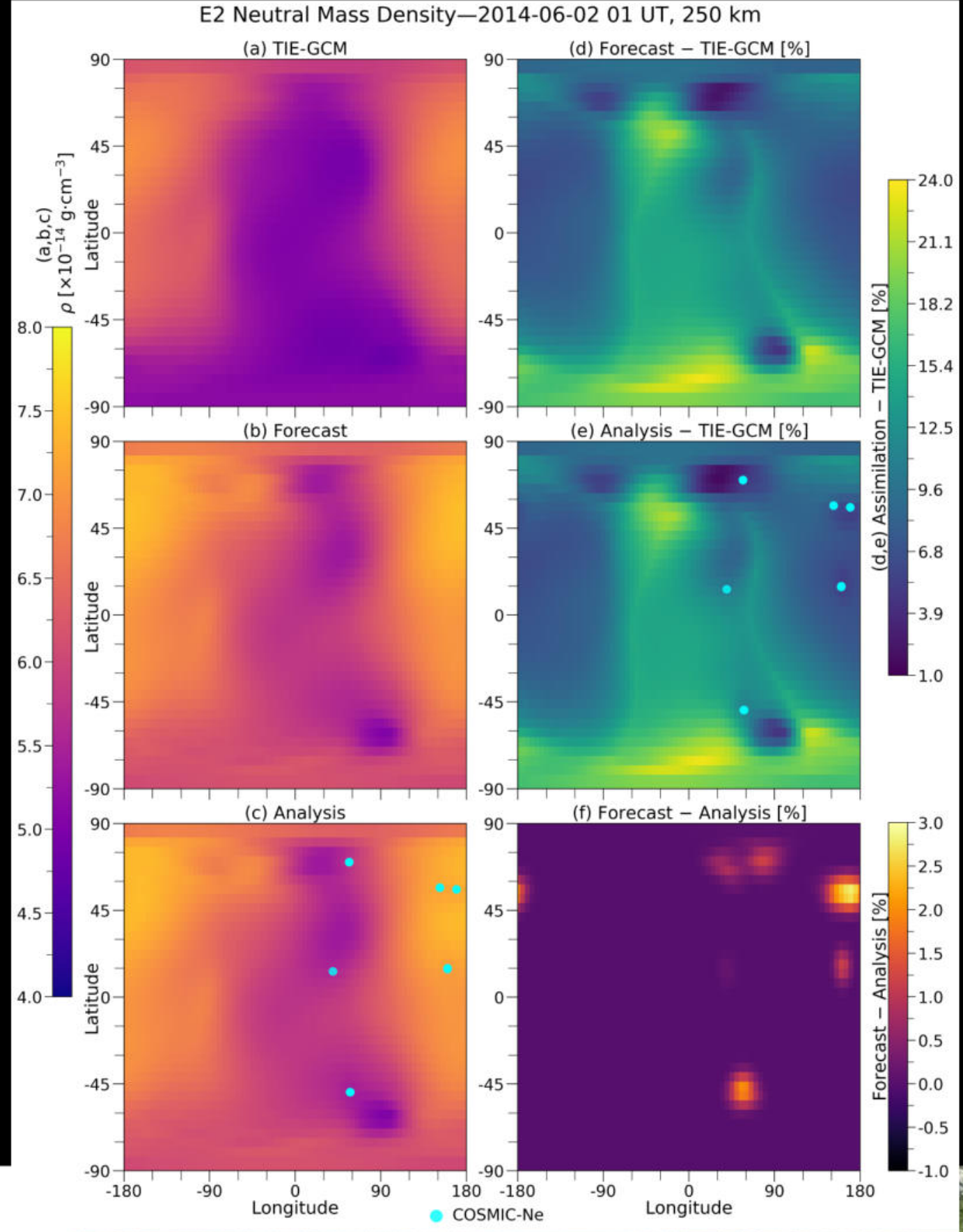


E2 Neutral Mass Density-2014-06-02 01 UT, 350 km
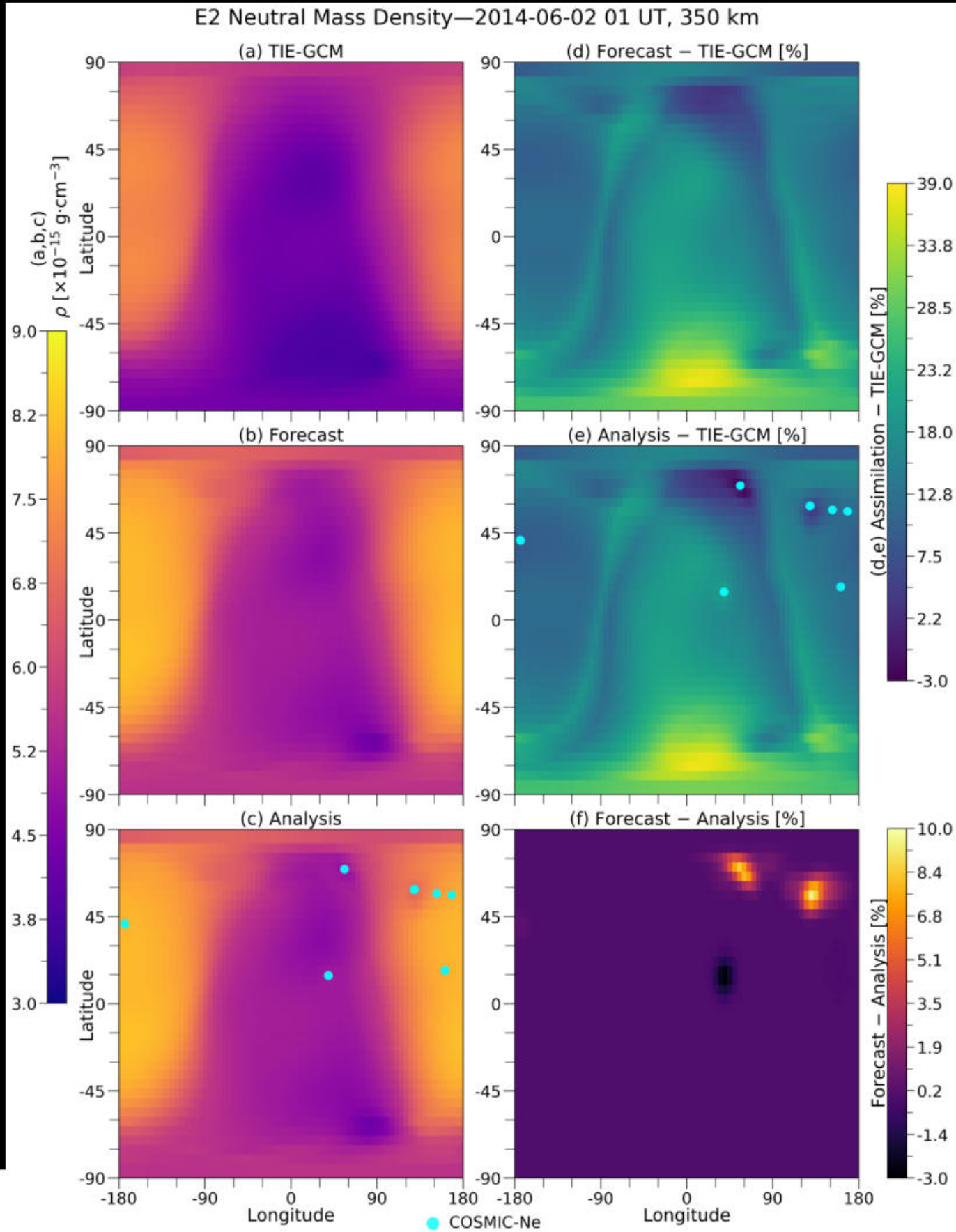
E2 Neutral Mass Density-2014-06-02 01 UT, 450 km

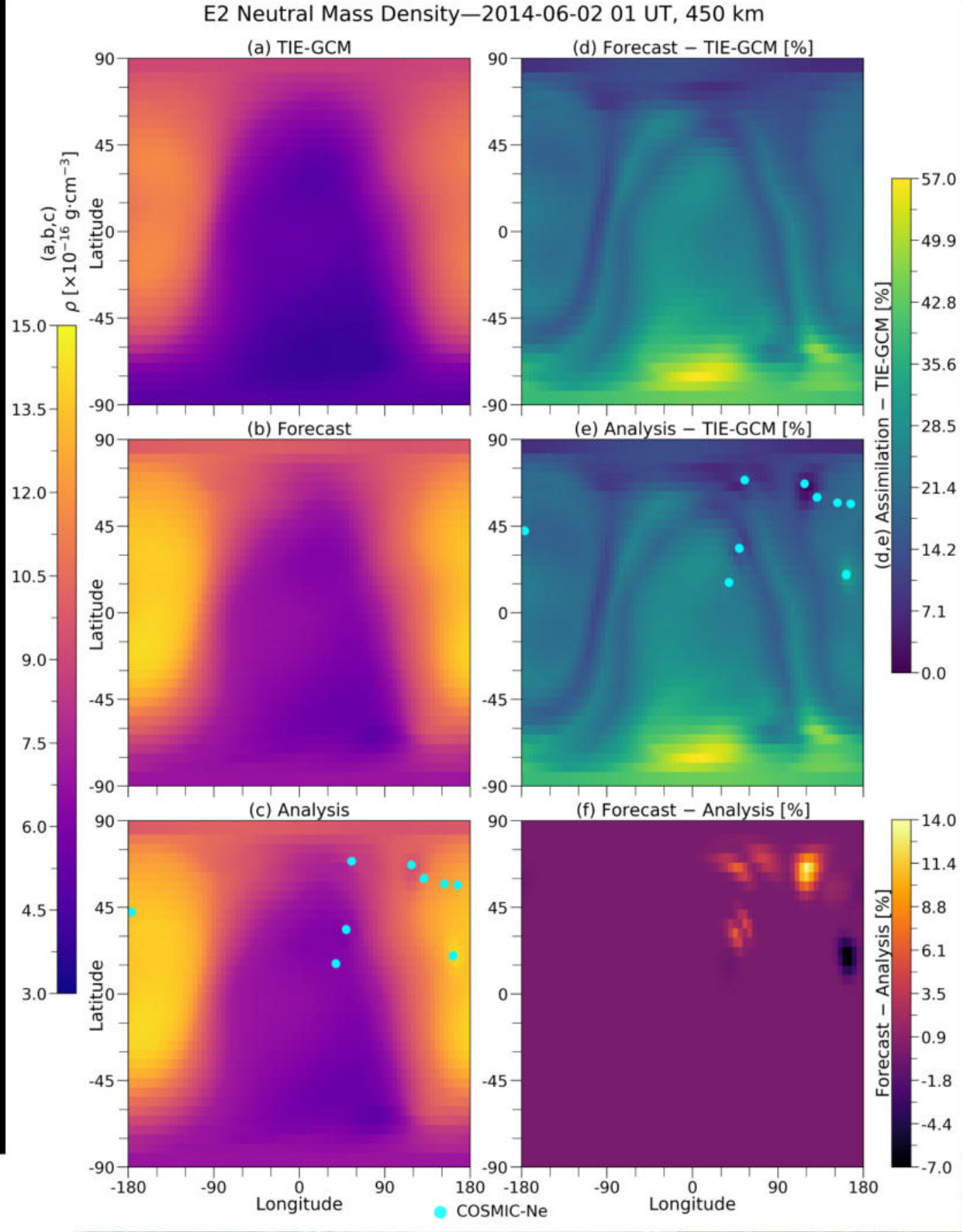


E2 Neutral Mass Density-2014-06-03 15 UT, 350 km
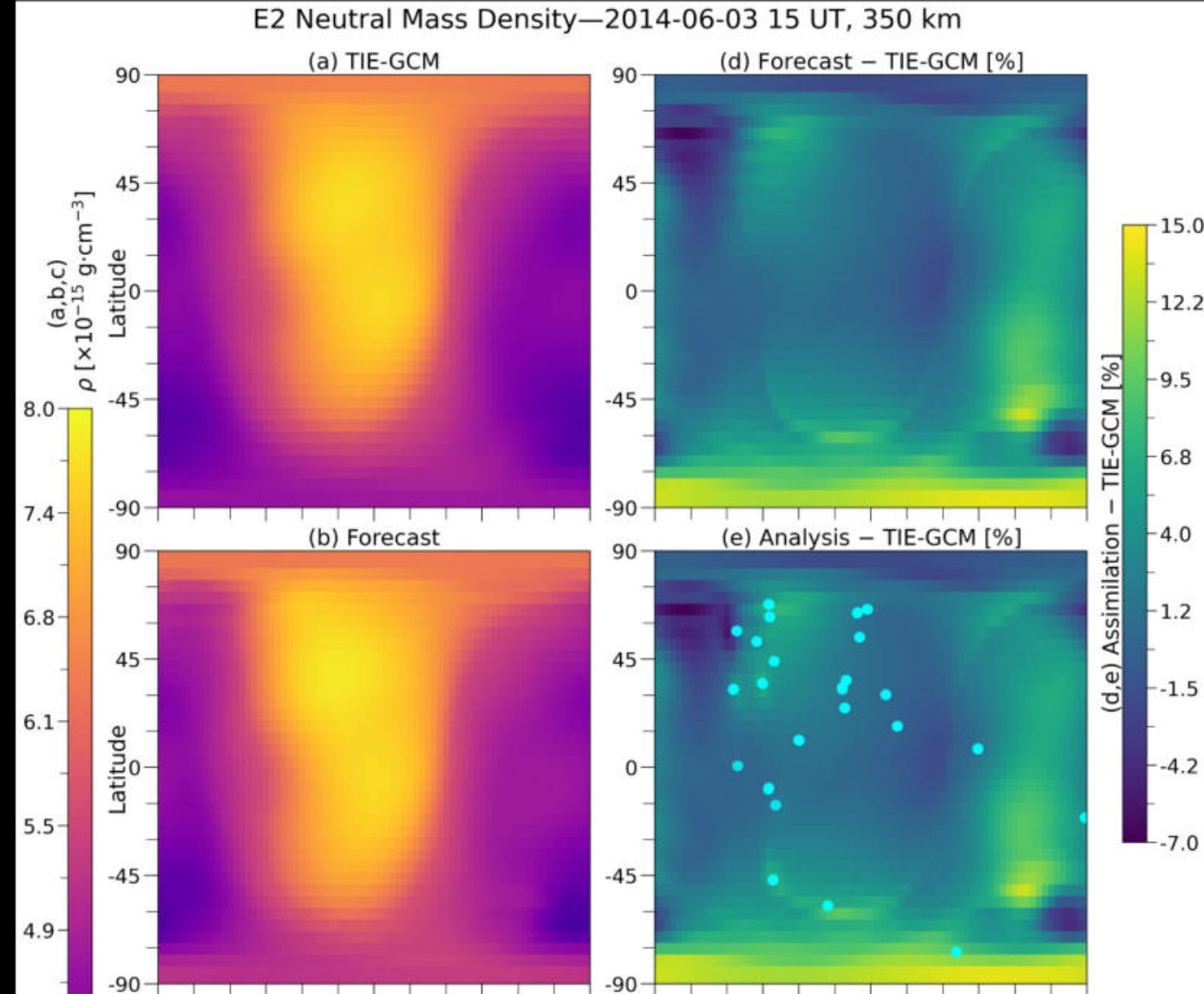

$3.6-$

(c) Analysis

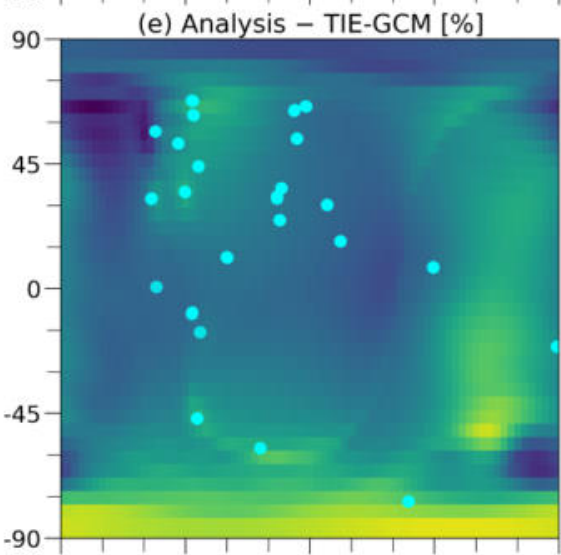

$-4.0$

(c) Analysis

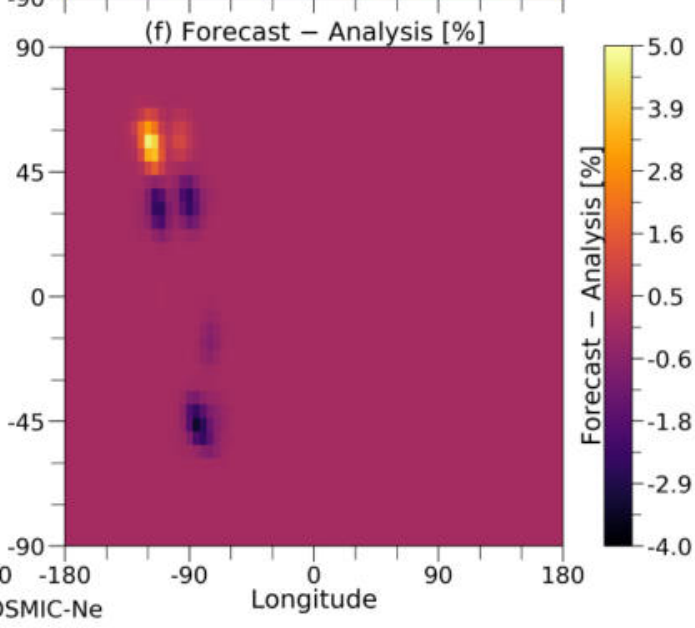

$\begin{array}{ll}90 & 180-90-180 \\ & \text { COSMIC-Ne }\end{array}$ 
E2 Neutral Mass Density-2014-06-03 15 UT, 450 km
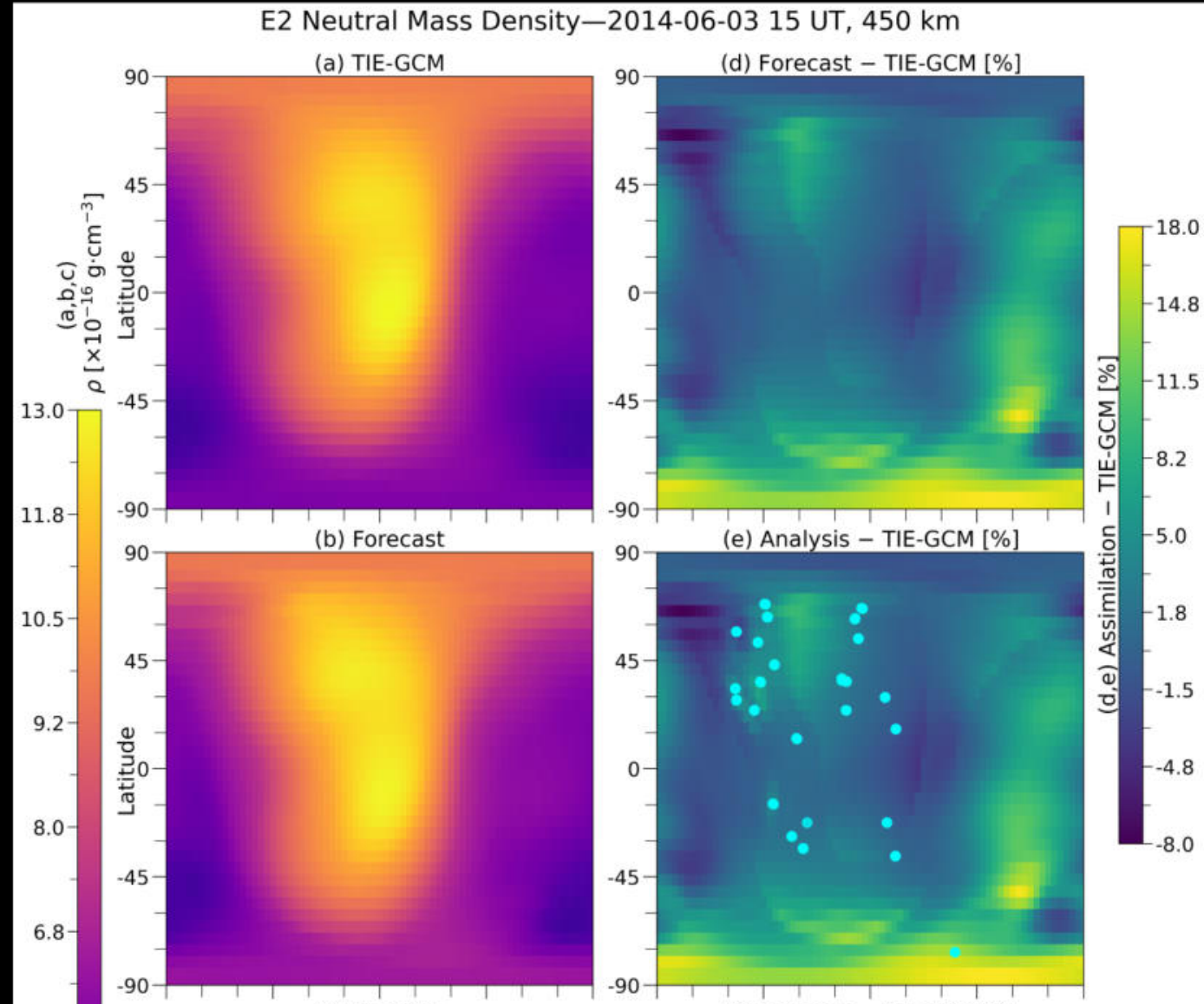

$-5.0$

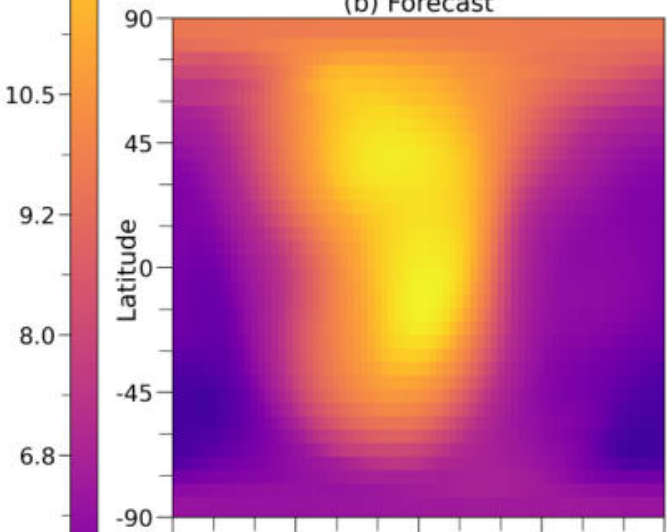

3.5-
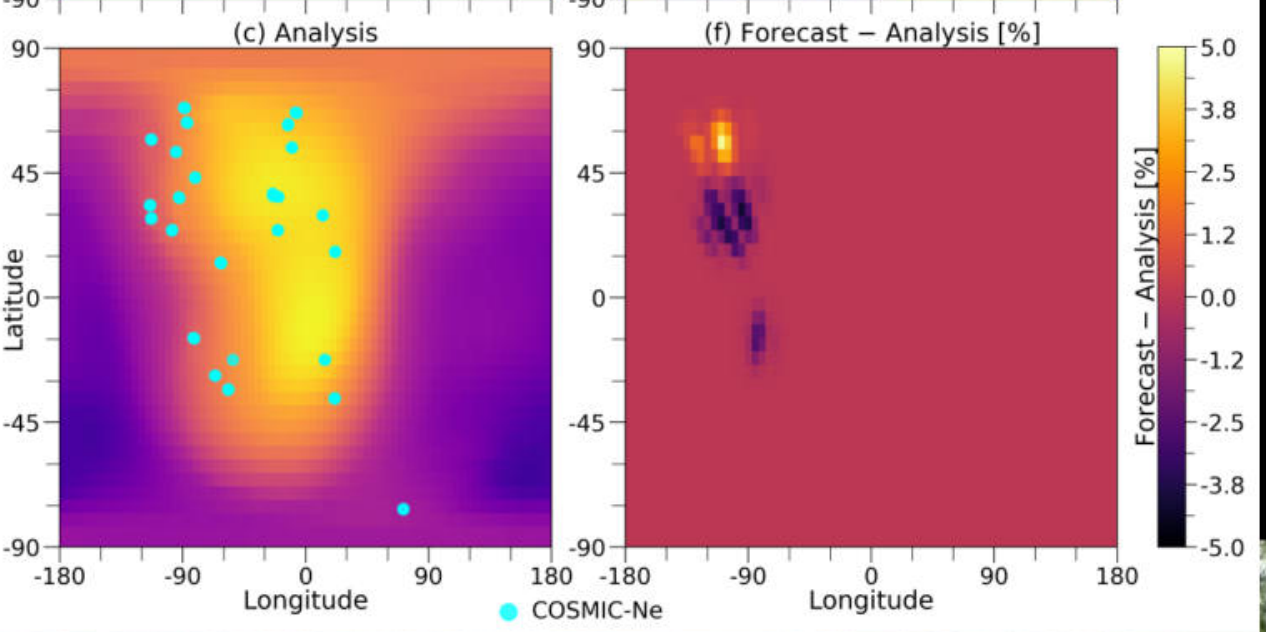
COSMIC-Ne 


\section{Summary and Conclusions}

Above results correspond to two experiments of assimilating electron densities into TIE-GCM using the EnKF technique. The results demonstrated the capability of the assimilation technique in the presence of realistic data assimilation scenarios to forecast the highly dynamical thermosphere.

The comparisons in the observation-space showed that assimilation of COSMIC-Ne can significantly change the model state to be inline with the observations. This change is more pronounced in the E2 night-time electron density profiles than others. The number of COSMIC-Ne profiles available to assimilate significantly depends on latitude and local time.

The validation results showed that the COSMIC-Ne-guided ionosphere state outperforms the GPI-guided TIE-GCM.

The results also demonstrated that assimilation of electron density can significantly impact the neutral mass density estimates of the model.

./continued 
The experiments E1 and E2 indicated that using COSMIC-Ne profiles in an operational-forecasting setting is challenging and that the area and local time coverages of the profiles are perhaps too sparse to be used in, for example, applications of orbit prediction.

The comparisons of neutral mass density may provide insights into the biases inherent in TIE-GCM-particularly along thermospheric features with sharp spatial gradients. The systematic biases that above results highlighted could be an indication that the specification of plasmaneutral interactions in TIE-GCM needs further adjustments.

The experiments mainly focused on the assimilation accuracy during different solar activity periods. More work needs to be done to identify and improve model bias due to external forcing. Assimilation of other thermospheric data, for example, atomic oxygen, thermospheric neutral winds and temperature could also help unravel some of the difficulties associated with forecasting thermospheric mass density. 


\section{References}

- Heelis, R. A., J. K. Lowell, and R. W. Spiro (1982), A model of the high-latitude ionospheric convection pattern, Journal of Geophysical Research: Space Physics, 87(A8), 6339-6345, doi:10.1029/JA087iA08p06339.

- Hagan, M. E., R. G. Roble, and J. Hackney (2001), Migrating thermospheric tides, Journal of Geophysical Research: Space Physics, 106(A7), 12,739-12,752, doi:10.1029/2000JA000344.

- Qian, L., S. C. Solomon, and T. J. Kane (2009), Seasonal variation of thermospheric density and composition, Journal of Geophysical Research: Space Physics, 114(A1), doi:10.1029/2008JA013643.

- Richmond, A. D., E. C. Ridley, and R. G. Roble (1992), A thermosphere/ionosphere general circulation model with coupled electrodynamics, Geophysical Research Letters, 19(6), 601-604, doi:10.1029/92GL00401.

- Solomon, S. C., and L. Qian (2005), Solar extreme-ultraviolet irradiance for general circulation models, Journal of Geophysical Research: Space Physics, 110(A10), doi:10.1029/2005JA011160. 